\title{
Direct numerical simulations of the $\kappa$-mechanism
}

\section{Radial modes in the purely radiative case}

\author{
T. Gastine and B. Dintrans
}

\author{
Laboratoire d'Astrophysique de Toulouse-Tarbes, Université de Toulouse, CNRS, 14 avenue Édouard Belin, 31400 Toulouse, France \\ e-mail: thomas.gastine@ast.obs-mip.fr
}

Received 26 October 2007 / Accepted 17 February 2008

\begin{abstract}
Context. We present a purely-radiative hydrodynamical model of the $\kappa$-mechanism that sustains radial oscillations in Cepheid variables.

Aims. We determine the physical conditions favourable for the $\kappa$-mechanism to occur inside a layer, with a configurable conductivityhollow. We complete nonlinear direct numerical simulations (DNS) that initiate from these most favourable conditions.

Methods. We compare the results of a linear-stability analysis, applied to radial modes using a spectral solver, and a DNS, which is developed from a high-order finite difference code.

Results. We find that by changing the location and shape of the hollow, we can generate well-defined instability strips. For a given position in the layer, the amplitude and width of the hollow appear to be key parameters to vary to attain unstable modes driven by the $\kappa$-mechanism. The DNS, starting from the favourable conditions, confirm both the growth rates and the structures of linearly-unstable modes. Nonlinear saturation is produced by intricate couplings between excited fundamental mode and higher damped overtones.
\end{abstract}

Key words. hydrodynamics - instabilities - waves - stars: oscillations - methods: numerical

\section{Introduction}

Since the first studies of Cepheid variables, it has been known that convection occurs inside their envelopes, which change their pulsation properties (e.g. the reviews of Gautschy \& Saio 1996; Buchler 1997). The coldest Cepheids, which are located close to the red edge of the instability strip, have the most extended surface convective zones. For many years, however, models of the oscillations of Cepheids have used the so-called "frozen-in convection" approximation, which assumes that convective flux perturbations are negligible (Baker \& Kippenhahn 1962). Such models reproduce the blue edge of the instability strip but not its red edge because, in this case, the strong existing couplings between convection and oscillations cannot be modelled accurately. This discrepancy is evident in high-quality observations that show a narrower instability strip than predicted theoretically, i.e. modes that are linearly-unstable in the models but located outside the observational strip (Yecko et al. 1998, hereafter YKB98).

The main theoretical difficulty is that convection plays a crucial role in pulsations, while we know that convection itself is roughly described by mixing-length theories (Vitense 1953; Böhm-Vitense 1958). However, several time-dependent convection models (TDC) have been developed (e.g. Unno 1967; Gough 1977; Stellingwerf 1982). Recent studies (YKB98; Bono et al. 1999) rely on Stellingwerf's convection model (Stellingwerf 1982) or similar more modern approaches to compute the linear and nonlinear time evolution of the amplitudes of modes (Kuhfuß 1986; Gehmeyr \& Winkler 1992; Wuchterl \& Feuchtinger 1998). The major problem of TDC is the choice of many free parameters introduced by the convection model ${ }^{1}$. As

${ }^{1}$ E.g. the seven coefficients $\left[\alpha_{\mathrm{c}}, \alpha_{\mathrm{t}}, \alpha_{v}, \alpha_{\Lambda}, \alpha_{\mathrm{s}}, \alpha_{\mathrm{d}}, \alpha_{\mathrm{p}}\right]$ in YKB98 or the eight ones in Kolláth et al. (2002). these parameters cannot be theoretically well-determined, they must be constrained by direct fitting of the observational data to the models.

Another way to study the convection-pulsation interaction is to develop (nonlinear) direct numerical simulations (DNS) of the entire hydrodynamical problem. We aim to develop such simulations in $2 \mathrm{D}$ and $3 \mathrm{D}$, in which a convective zone is coupled with a radiative one and unstable radial acoustic modes are self-consistently excited by the $\kappa$-mechanism. However, as DNS are highly time-consuming, it is necessary to use the appropriate initial conditions. In this paper, we attempt to determine the physical conditions that are favourable to excitation based on the $\kappa$-mechanism.

Eddington (1917), and then Zhevakin (1953) and Cox (1958) introduced a mechanism linked to opacity in ionisation regions, the $\kappa$-mechanism, where $\kappa$ denotes opacity (see also the review of Zhevakin 1963). They showed that Cepheids' radial oscillations are driven by a thermal heat engine, since radial pulsations must be maintained by a sustained physical process. The process they proposed was a Carnot-like thermodynamic cycle, which stores heat during compression phases and releases heat during decompression. This mechanism, now referred to as the Eddington's valve, can occur only in regions of a star where the opacity varies such that the radiative flux is blocked during compression phases. Opacity tables such as OPAL show strong increases in ionisation regions of main (i.e. hydrogen and helium) or heavy elements that are commonly called "ionisation bumps" (e.g. Seaton \& Badnell 2004). As a consequence, these ionisation zones are locally responsible for mode amplification. Apart from this restriction on opacity, these ionisation regions have to be located in a precise region of the star. Indeed, if they are too deep or too close to the surface, the driving they cause can be balanced by the damping occurring in other regions. Therefore, 
an efficient ionisation region has to be located in a specific place, called the transition region, which marks the shift from a quasiadiabatic interior to a strongly non-adiabatic surface. For classical Cepheids that oscillate in the radial fundamental mode, the temperature overlap of the transition with the ionisation region is about $40000 \mathrm{~K}$, which corresponds to the temperature of helium second ionisation (Baker \& Kippenhahn 1965). For first overtone Cepheids, the processes are more delicate because we must consider firstly the respective positions of the HeII ionisation region and nodal line, and secondly the $\mathrm{HeI} / \mathrm{H}$ region, which contributes to driving (Bono et al. 1999).

However, the location of these opacity bumps are not solely responsible for acoustic-mode destabilisation. A careful treatment of the $\kappa$-mechanism in Cepheids would involve the possible dynamical couplings with convective zones or, perhaps, metallicity effects, which would be described by a realistic equation of state and opacity tables. The corresponding physics is not fully understood theoretically and the purpose of our work is to simplify the hydrodynamical approach while retaining the leading order phenomenon responsible for the instability, that is, the opacity bumps location.

We therefore adopt a fully radiative layer of a perfect gas in which an ionisation region is represented by a "hollow" in radiative conductivity, corresponding to a "bump" in opacity. The layer stability should depend on both temperature and density variations, because the radiative conductivity is a function of both physical quantities. However, since the opacity depends strongly on temperature, this state variable mainly controls the instability. We propose to develop a hydrodynamical study of the $\kappa$-mechanism and therefore neglect any dependence of radiative conductivity on density; our conductivity profile is therefore a function of temperature alone. This allows easy changes in the different parameters of the ionisation region by setting both position and shape of the conductivity hollow (i.e. its slope, width and amplitude). It is therefore possible to investigate a complete parametric study of $\kappa$-mechanism to determine precisely the physical conditions required for the instability to occur.

We first consider the radiative and hydrostatic equilibria of our layer with an appropriate conductivity profile. By adjusting both the density value at the top of the layer and the flux at its bottom, we obtain a transition region in the middle of the computational domain. Secondly, we investigate the linear-stability analysis by solving perturbation equations for the oscillations. We obtain the entire spectrum, and are able to distinguish unstable modes from stable ones. We are therefore able to check the relevance of the transition region concept by making every parameters of the conductivity hollow vary.

The first main result of our linear-stability study is the confirmation of the underlying conditions defining the transition region. With different parametric studies, we obtain instability strips corresponding to the fundamental mode because both a minimum hollow width and amplitude are needed to obtain unstable modes. We interpret these results using the work integral to determine the precise location of the driving zone. This parametric study of the $\kappa$-mechanism provides the physical quantities that create the instability.

The favourable conditions required to excite the fundamental mode form the initial conditions of the 2D DNS. We monitor the growth rates and structure of the linearly-unstable modes by completing direct simulations until nonlinear saturation of modes occurs.

In Sect. 2, we first introduce the general oscillation equations. The different conditions leading to the instability are determined in Sect. 3. In Sect. 4, we present our hydrodynamical

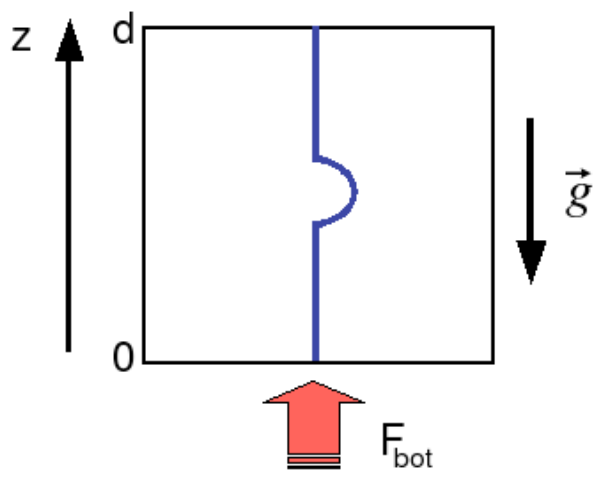

Fig. 1. Scheme corresponding to our model. Gravity is pointing downward, in the opposite direction to the vertical coordinate. The (blue) curve represents the radiative conductivity profile that we discuss further, and the large (red) arrow represents the radiative flux entering the bottom of the layer.

model. Linear stability analysis and DNS results are provided and compared in Sects. 5 and 6, respectively. We outline our conclusion in Sect. 7.

\section{The general oscillations equations}

Our system is composed of a 2D plane parallel layer of width $d$. $z$ denotes a Cartesian coordinate, pointing upward in contrast to the constant gravity field $\boldsymbol{g}$. The gas is assumed to be monatomic and perfect, and its equation of state is given by

$p=R_{*} \rho T \quad$ and $\quad \gamma \equiv \frac{c_{\mathrm{p}}}{c_{\mathrm{v}}}=\frac{5}{3}$,

where $p, \rho$ and $T$ respectively denote pressure, density and temperature, $R_{*}$ is the ideal gas constant, and $\gamma$ is the ratio of specific heats $c_{\mathrm{p}}$ and $c_{\mathrm{v}}$.

We are interested in small perturbations about an equilibrium state, that is, any physical quantity is expanded about its mean value $X_{0}(\boldsymbol{r})$ in the following way

$X(\boldsymbol{r}, t)=X_{0}(\boldsymbol{r})+X^{\prime}(\boldsymbol{r}, t) \quad$ with $\quad X^{\prime} / X_{0} \ll 1$,

where $X^{\prime}$ is an Eulerian perturbation. Linearised continuity, momentum and energy equations for the perturbations in a nonadiabatic case, are provided by (e.g. Unno et al. 1989)

$$
\left\{\begin{aligned}
\lambda \rho^{\prime}= & -\rho_{0} \operatorname{div} \boldsymbol{u}-\boldsymbol{u} \cdot \boldsymbol{\nabla} \ln \rho_{0}, \\
\lambda \boldsymbol{u}= & -\frac{1}{\rho_{0}} \boldsymbol{\nabla} p^{\prime}+\frac{\rho^{\prime}}{\rho_{0}} \boldsymbol{g} \\
& +v\left(\Delta \boldsymbol{u}+\frac{1}{3} \boldsymbol{\nabla} \operatorname{div} \boldsymbol{u}+2 \boldsymbol{S} \cdot \boldsymbol{\nabla} \ln \rho_{0}\right), \\
\lambda T^{\prime}= & -\frac{1}{\rho_{0} c_{\mathrm{v}}} \operatorname{div} \boldsymbol{F}^{\prime}-(\gamma-1) T_{0} \operatorname{div} \boldsymbol{u}-\boldsymbol{u} \cdot \boldsymbol{\nabla} T_{0},
\end{aligned}\right.
$$

where $\boldsymbol{u}, \rho^{\prime}, T^{\prime}$ and $\boldsymbol{F}^{\prime}$ denote the velocity, density, temperature and radiative flux perturbations, respectively. The kinematic viscosity $v$ is supposed to be constant and $\boldsymbol{S}$ denotes the traceless rate-of-strain tensor given by

$S_{i j}=\frac{1}{2}\left(\frac{\partial u_{i}}{\partial x_{j}}+\frac{\partial u_{j}}{\partial x_{i}}-\frac{2}{3} \delta_{i j} \operatorname{div} \boldsymbol{u}\right)$ 
We seek normal modes with a time-dependence of the form $\exp (\lambda t)$, where $\lambda=\tau+\mathrm{i} \omega$. The real part $\tau$ denotes the growth $(\tau>0)$ or damping $(\tau<0)$ rate whereas the imaginary part $\omega$ denotes the frequency. We assume that the layer is fully radiative, that under the diffusion approximation, leads to the following expression for the radiative flux perturbation

$\boldsymbol{F}^{\prime}=-K_{0} \boldsymbol{\nabla} T^{\prime}-K^{\prime} \boldsymbol{\nabla} T_{0}$,

where $K_{0}$ denotes the radiative conductivity and $K^{\prime}$ its Eulerian perturbation. This perturbation $K^{\prime}$ can be related in a general way to that of the temperature using the relations

$\frac{K^{\prime}}{K_{0}}=\mathcal{K}_{T} \frac{T^{\prime}}{T_{0}} \quad$ with $\quad \mathcal{K}_{T}=\frac{\mathrm{d} \ln K_{0}}{\mathrm{~d} \ln T_{0}}$.

Finally, we impose the following boundary conditions

$$
\left\{\begin{array}{l}
u_{z}=0 \text { for } z=[0, d] \\
\frac{\mathrm{d} T^{\prime}}{\mathrm{d} z}=0 \text { for } z=0 \text { and } T^{\prime}=0 \text { for } z=d,
\end{array}\right.
$$

which correspond to rigid walls at both limits of the domain, a perfect conductor at the bottom, and a perfect insulator at the top.

\section{Conditions for instability}

\subsection{A first condition derived from the work integral}

We recall that the principle aim of our work is to clarify the favourable conditions that can sustain unstable radial modes in a plane-parallel layer. A helpful way to study the physics of such an instability is the use of the work integral. In the following, we assume that transformations are quasi-adiabatic. This approximation is sufficient in the deeper layers of a star but becomes no longer valid close to its surface where non-adiabatic effects dominate. This approximation is, however, useful for study of the stability properties of an oscillation mode.

Using the work-integral formalism in the quasi-adiabatic limit, we demonstrate in Appendix A.1 the following expression for the damping or growth rate of an eigenmode

$\tau=-\frac{\mathfrak{R}\left[\int_{V}(\gamma-1) \frac{\delta \rho^{*}}{\rho} \operatorname{div} \boldsymbol{F}^{\prime} \mathrm{d} V\right]}{\int_{V}|\boldsymbol{u}|^{2} \rho_{0} \mathrm{~d} V}$,

where the symbol $\mathfrak{R}$ represents the real part. We can see that the sign of $\tau$ only depends on the numerator, which, under the diffusion approximation, Eq. (5), leads to

$\tau \propto \mathfrak{R}\left[\int_{0}^{d} \frac{\delta \rho^{*}}{\rho_{0}} \operatorname{div}\left(K^{\prime} \boldsymbol{\nabla} T_{0}+K_{0} \boldsymbol{\nabla} T^{\prime}\right) \mathrm{d} z\right]$.

The main driving term in this expression is $K^{\prime} \nabla T_{0}$ because it represents the dynamical variation of opacity during an oscillation cycle, which is the cause of the $\kappa$-mechanism. We neglect $K_{0} \nabla T^{\prime}$ to obtain

$\tau \propto \mathfrak{R}\left[\int_{0}^{d} \frac{\delta \rho^{*}}{\rho_{0}} \operatorname{div}\left(K^{\prime} \nabla T_{0}\right) \mathrm{d} z\right]$.

As we can see in Fig. 3b, the equilibrium temperature is an almost linear function of $z$, except in the vicinity of the conductivity hollow. $\boldsymbol{\nabla} T_{0}$ is therefore almost constant and we have

$\tau \propto \mathfrak{R}\left[\int_{0}^{d} \frac{\delta \rho^{*}}{\rho_{0}} \boldsymbol{\nabla} T_{0} \boldsymbol{\nabla} K^{\prime} \mathrm{d} z\right]$.
Using Eq. (6), we obtain $K^{\prime}=K_{0}\left(T_{0}\right) \mathcal{K}_{T} T^{\prime} / T_{0}$. We assume that $\boldsymbol{\nabla} K^{\prime}$ is dominated by $K_{0}\left(T_{0}\right) T^{\prime} / T_{0} \nabla \mathcal{K}_{T}$ because we are interested in low-order modes. We then obtain

$\tau \propto \mathfrak{R}\left[\int_{0}^{d} \frac{\delta \rho^{*}}{\rho_{0}} \nabla T_{0} K_{0}\left(T_{0}\right) \frac{T^{\prime}}{T_{0}} \nabla \mathcal{K}_{T} \mathrm{~d} z\right]$.

We now consider a compression phase corresponding to $\delta \rho^{*} / \rho_{0}>0$ and $T^{\prime} / T_{0}>0$. Since $\nabla T_{0}<0$, a necessary condition to obtain unstable modes with $\tau>0$ is

$\frac{\mathrm{d} \mathcal{K}_{T}}{\mathrm{~d} z}<0$.

In variable stars, this condition occurs in ionisation regions. Opacity tables show clearly that these regions are associated with large "bumps" in opacity (e.g. the review of Carson 1976; Seaton \& Badnell 2004). Using Eq. (13), we can obtain unstable modes if driving prevails over damping. This result implies that if the radiative conductivity decreases during a compression phase, then some part of the radiative flux is blocked; some energy is then stored during compression, which contributes to an increase in the ratio of ionised matter. During the decompression phase, this extra energy is released, by means of mechanical work, to the environment, and can excite oscillations. This operates in a similar way to the thermal heat engine that Eddington proposed to explain the oscillations of Cepheids (Eddington 1917).

\subsection{A second condition derived from the so-called "transition region"}

In the previous paragraph, we recalled a necessary condition to obtain unstable modes (Eq. (13)). Nevertheless, the demonstration was made under the quasi-adiabatic approximation which fails close to the surface. We are therefore unable to tell if driving caused by ionisation regions prevails over other damping regions. Ionisation regions, for which Eq. (13) is satisfied, are thin compared to the entire stellar atmosphere; the influence of damping regions on instability therefore remains questionable. Hereafter, we follow Cox's demonstration (e.g. Cox 1980; Christensen-Dalsgaard 2003).

In Lagrangian variables, the energy equation can be written as

$\frac{\mathrm{d}}{\mathrm{d} t}\left(\frac{\delta T}{T_{0}}\right)-(\gamma-1) \frac{\mathrm{d}}{\mathrm{d} t}\left(\frac{\delta \rho}{\rho_{0}}\right)=-\frac{F_{\mathrm{bot}}}{\rho_{0} c_{\mathrm{v}} T_{0}} \operatorname{div}\left(\frac{F^{\prime}}{F_{\text {bot }}}\right)$,

where the symbol $\delta$ means Lagrangian perturbations. Since we are considering a $1 \mathrm{D}$ box, we have

$-d\left(\frac{F^{\prime}}{F_{\text {bot }}}\right) \mathrm{d} t=\frac{\rho_{0} c_{\mathrm{v}} T}{F_{\text {bot }}} d\left[\frac{\delta T}{T_{0}}-(\gamma-1) \frac{\delta \rho}{\rho_{0}}\right] \mathrm{d} z$.

We integrate between a given height $z$ and the surface, to obtain

$\Delta\left(\frac{F^{\prime}}{F_{\text {bot }}}\right) \Pi \sim \frac{\left\langle c_{\mathrm{v}} T_{0}\right\rangle}{F_{\text {bot }}} \int_{z}^{d} \rho_{0} \mathrm{~d} z^{\prime} d\left[\frac{\delta T}{T_{0}}-(\gamma-1) \frac{\delta \rho}{\rho_{0}}\right]$,

where $\Delta\left(F^{\prime} / F_{\text {bot }}\right)$ is the variation of $F^{\prime} / F_{\text {bot }}$ between the considered point and the surface, $\left\langle c_{\mathrm{v}} T_{0}\right\rangle$ is an average of $c_{\mathrm{V}} T_{0}$ over this region, and $\Pi$ is the characteristic dynamical timescale, which is typically the pulsation period of the fundamental mode. Since we are principally interested in the study of low-order modes (i.e. the fundamental one), we assume, as in Eq. (12), that the eigenvectors are almost constant (e.g. Cox 1980). We then obtain

$d\left[\frac{\delta T}{T_{0}}-(\gamma-1) \frac{\delta \rho}{\rho_{0}}\right] \approx\left\langle\frac{\delta T}{T_{0}}-(\gamma-1) \frac{\delta \rho}{\rho_{0}}\right\rangle_{z}$, 
where $\langle.\rangle_{z}$ represents the average of an eigenfunction. We note that

$$
\int_{z}^{d} \rho_{0} \mathrm{~d} z^{\prime}=\frac{\Delta m}{S} \quad \text { and } \quad L=F_{\text {bot }} S,
$$

leads to

$\Delta\left(\frac{F^{\prime}}{F_{\text {bot }}}\right) \sim \Psi\left\langle\frac{\delta T}{T_{0}}-(\gamma-1) \frac{\delta \rho}{\rho_{0}}\right\rangle_{z}$,

where

$\Psi=\frac{\left\langle c_{\mathrm{v}} T_{0}\right\rangle \Delta m}{\Pi L}$.

$\Psi$ represents the ratio of the thermal energy embedded between the considered point and the surface to the energy radiated during an oscillation period ${ }^{2}$. A second instability criterion can be derived from the value of this quantity $\Psi$ :

- If the considered point is too deep inside the star, the local thermal timescale is longer than the dynamical one, and $\Psi \gg$ 1. Pulsation has therefore no influence on the energy release and the adiabatic approximation ${ }^{3}$ is well-suited in this case.

- In contrast, if the considered point is close to the surface, then $\Delta m$ is small and $\Psi \ll 1$. The energy content (and the corresponding mass content) is then too weak to influence the radiative flux and the luminosity perturbation is frozenin. The radiative flux perturbation does not vary further in this region.

- Between these two regions, $\Psi$ can be $O(1)$, which defines a zone called the transition region, that separates the adiabatic interior from the strongly non-adiabatic surface, that is,

$\Psi=\frac{\left\langle c_{\mathrm{V}} T_{0}\right\rangle \Delta m}{\Pi L} \sim 1$.

King \& Cox (1968) first determined the temperature associated with this transition region for different Cepheid models. For the fundamental mode, they derived $T_{\mathrm{TR}} \simeq 40000 \mathrm{~K}$, while the transition was closer to the surface for higher-order modes.

The corresponding positions of both ionisation and transition regions are crucial for instability to occur (Gilliland et al. 1998). If the regions overlap, which occurs in the instability strip, the bottom of the ionisation region contributes strongly to driving because it acts under quasi-adiabatic conditions. In contrast, its top is in a strongly non-adiabatic region where the luminosity is frozen-in. The zone that is over the ionisation region is then not affected by damping and driving prevails, that is, modes become unstable (see e.g. Cox 1980, for a more detailed description).

We present the conditions favourable to instability to be

$$
\frac{\mathrm{d} \mathcal{K}_{T}}{\mathrm{~d} z}<0 \quad \text { and } \quad T_{\mathrm{TR}} \sim T_{\text {ionisation }}
$$

Using the conditions provided in Eq. (22), we obtain $T_{\text {ionisation }} \simeq$ $40000 \mathrm{~K}$. This temperature corresponds to the second Helium ionisation, which is known to be responsible primarily for the driving of modes in Cepheids (see Cox 1963).

\footnotetext{
$2 \Psi$ can also be interpreted as the ratio of the local thermal timescale to the dynamical one.

3 One can notice that the "adiabatic" approximation means here that deep inside the layer the mode has an adiabatic behaviour whereas close to the surface it remains strongly non-adiabatic.
}

\section{Our hydrodynamical model}

\subsection{The choice of the radiative conductivity profile}

Radiative conductivity $K_{0}$ depends on both temperature and density because the diffusion approximation provides (e.g. Mihalas \& Weibel Mihalas 1984)

$K_{0}=\frac{16 \tilde{\sigma} T^{3}}{3 \kappa \rho}$

where $\tilde{\sigma}$ denotes Stefan-Boltzmann's constant. Kramer's laws approximate the opacity laws with

$\kappa \propto \rho^{n} T^{-s}$,

where, for example, $n=1$ and $s=3.5$ for the free-free opacity (e.g. Hansen \& Kawaler 1994). As shown in the Introduction and in Sect. 2, we consider a constant radiative conductivity, upon which a hollow representing a bump in opacity is added. This simple approach is an easy way to reproduce the physical conditions favourable to the $\kappa$-mechanism, while allowing the hollow amplitude, slope or width to be quickly changed; we then achieve an efficient parametric study of the instability. The radiative conductivity is then given by ${ }^{4}$

$K_{0}(T)=K_{\max }\left[1+\mathcal{A} \frac{-\pi / 2+\arctan \left(\sigma T^{+} T^{-}\right)}{\pi / 2+\arctan \left(\sigma e^{2}\right)}\right]$,

with

$\mathcal{A}=\frac{K_{\max }-K_{\min }}{K_{\max }}, \quad T^{ \pm}=T-T_{\text {bump }} \pm e$,

where

- $T_{\text {bump }}$ is the position of the hollow in temperature;

- $K_{\max }$ and $K_{\min }$ are the conductivity extrema and $\mathcal{A}$ is the corresponding relative amplitude;

- $\sigma$ represents the slope of the hollow;

$-e$ represents the half of the full width at half maximum (i.e. $F W H M / 2)$ of the hollow.

Examples of common values of these parameters are provided in Fig. 2.

\subsection{The equilibrium setup}

Hydrostatic and radiative equilibria in the diffusion limit are given by

$\left\{\begin{array}{l}\boldsymbol{\nabla} p_{0}=\rho_{0} \boldsymbol{g}, \\ \operatorname{div}\left[K_{0}\left(T_{0}\right) \boldsymbol{\nabla} T_{0}\right]=0 .\end{array}\right.$

Assuming a constant radiative flux $F_{\text {bot }}$ at the bottom of the layer, implies that

$$
\left\{\begin{array}{l}
\frac{\mathrm{d} p_{0}}{\mathrm{~d} z}=-\rho_{0} g, \\
\frac{\mathrm{d} T_{0}}{\mathrm{~d} z}=-\frac{F_{\text {bot }}}{K_{0}} .
\end{array}\right.
$$

\footnotetext{
4 This arctan-profile may appear quite intricate, compared e.g. to a Gaussian-like one, but it allows us to change each of the hollow parameters almost independently (i.e. changing its amplitude while keeping its width).
} 


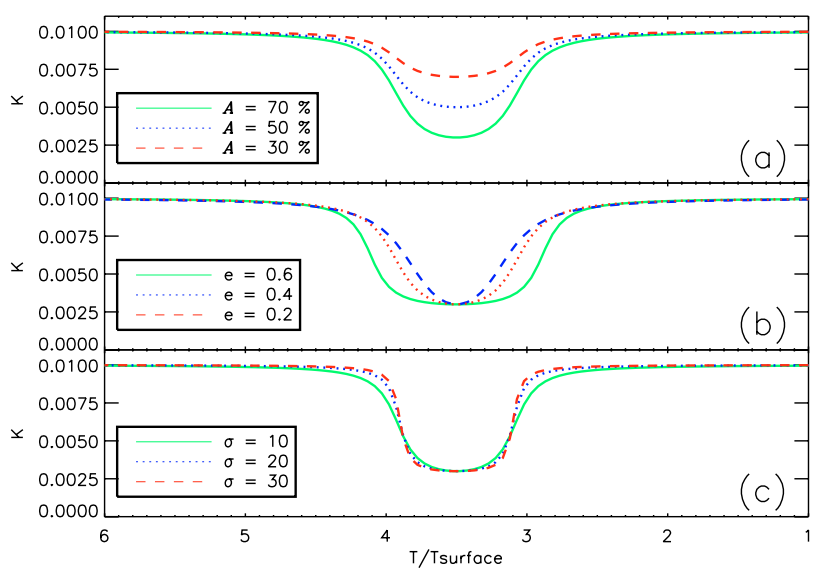

Fig. 2. Influence of the hollow parameters on the conductivity profile for $K_{\max }=10^{-2}$ and $T_{\text {bump }}=3.5$ : amplitude $\mathcal{A}(\mathbf{a})$, width $e(\mathbf{b})$ and slope $\sigma(\mathbf{c})$ (here the abscissa denotes a dimensionless temperature based on the surface one, i.e. $T / T_{\text {surface }}$ ).

We select the depth of the layer $d$ to be the length scale, and the temperature at the top of the layer $T_{\text {top }}$ to be the temperature scale, i.e. $[z]=d$ and $[T]=T_{\text {top }}$. The top density is chosen as the density scale $\left([\rho]=\rho_{\text {top }}\right)$, velocity is provided in units of $\sqrt{c_{\mathrm{p}} T_{\text {top }}}$, gravity in units of $c_{\mathrm{p}} T_{\text {top }} / d$, pressure in units of $\rho_{\text {top }}\left[u^{2}\right]$, while radiative conductivity is provided in units of $\rho_{\text {top }} c_{\mathrm{p}} d[u]$. The corresponding radiative flux unit is then $\rho_{\text {top }}\left(c_{\mathrm{p}} T_{\text {top }}\right)^{3 / 2}$. The dimensionless equations become

$$
\left\{\begin{array}{l}
\frac{\mathrm{d} \ln \tilde{p}_{0}}{\mathrm{~d} z}=-\frac{\gamma}{\gamma-1} \frac{\tilde{g}}{\tilde{T}_{0}}, \\
\frac{\mathrm{d} \tilde{T}_{0}}{\mathrm{~d} z}=-\frac{\tilde{F}_{\text {bot }}}{\tilde{K}_{0}}, \\
\tilde{p}_{0}=\frac{\gamma-1}{\gamma} \tilde{\rho}_{0} \tilde{T}_{0} .
\end{array}\right.
$$

The set of Eqs. (29) can be written in matrix form as

$A \psi=B(\psi)$,

where $\psi=\left(p_{0}, T_{0}\right)^{T}$ is the equilibrium field vector, and $A, B$ are differential operators. We note that the RHS, $B(\psi)$, depends on the eigenvector itself through the terms $1 / \tilde{T}_{0}$ and $1 / \tilde{K}_{0}\left(\tilde{T}_{0}\right)$, which means solving a nonlinear problem.

Tildes on equilibrium fields parameters denote dimensionless quantities, which will be dropped for clarity in the following.

\subsection{About Schwartzschild's criterion in our model}

It is important to ensure that our box is entirely radiative. We must therefore adopt Schwartzschild's criterion (e.g. Chandrasekhar 1961)

$\left|\frac{\mathrm{d} T_{0}}{\mathrm{~d} z}\right|<\left|\frac{\mathrm{d} T_{0}}{\mathrm{~d} z}\right|_{\text {adia }} \quad$ with $\quad\left|\frac{\mathrm{d} T_{0}}{\mathrm{~d} z}\right|_{\text {adia }}=\frac{g}{c_{\mathrm{p}}}$.

If this inequality is ensured, then the layer is fully radiative. In our dimensionless units, this condition becomes

$\left|\frac{\mathrm{d} T_{0}}{\mathrm{~d} z}\right|<g$,

which implies that large variations in temperature throughout the domain require large values of the dimensionless gravity field $g$.

\subsection{Back to the radial oscillations equations}

To simplify the general system-of-oscillations Eqs. (3), we define the following new variables

$R \equiv \rho^{\prime} / \rho_{0} \quad$ and $\quad \theta \equiv T^{\prime} / T_{0}$

Using Eq. (29) and substituting the conductivity into the energy equation (see Appendix B), we obtain

$$
\left\{\begin{aligned}
\lambda R= & -\operatorname{div} \boldsymbol{u}-\boldsymbol{u} \cdot \boldsymbol{\nabla} \ln \rho_{0}, \\
\lambda \boldsymbol{u}= & -\frac{p_{0}}{\rho_{0}} \boldsymbol{\nabla}(\theta+R)-\theta \boldsymbol{g} \\
& +v\left(\Delta \boldsymbol{u}+\frac{1}{3} \boldsymbol{\nabla} \operatorname{div} \boldsymbol{u}+2 \boldsymbol{S} \cdot \boldsymbol{\nabla} \ln \rho_{0}\right), \\
\lambda \theta= & \frac{1}{\rho_{0} c_{\mathrm{v}} T_{0}} \Delta_{z}\left(K_{0} T_{0} \theta\right)-(\gamma-1) \operatorname{div} \boldsymbol{u}-\boldsymbol{u} \cdot \boldsymbol{\nabla} \ln T_{0} .
\end{aligned}\right.
$$

We then adopt the same dimensionless quantities used in the equilibrium Eqs. (29)

$$
\left\{\begin{aligned}
\lambda R= & -\frac{\mathrm{d} u_{z}}{\mathrm{~d} z}-\frac{\mathrm{d} \ln \rho_{0}}{\mathrm{~d} z} u_{z}, \\
\lambda u_{z}= & -\frac{\gamma-1}{\gamma} T_{0}\left(\frac{\mathrm{d} R}{\mathrm{~d} z}+\frac{\mathrm{d} \theta}{\mathrm{d} z}\right)+g \theta+\mathcal{D}_{v} \\
\lambda \theta= & \gamma \chi_{0}\left[\frac{\mathrm{d}^{2} \theta}{\mathrm{d} z^{2}}+2\left(\frac{\mathrm{d} \ln K_{0}}{\mathrm{~d} z}+\frac{\mathrm{d} \ln T_{0}}{\mathrm{~d} z}\right) \frac{\mathrm{d} \theta}{\mathrm{d} z}\right. \\
& \left.+\frac{\mathrm{d} \ln T_{0}}{\mathrm{~d} z} \frac{\mathrm{d} \mathcal{K}_{T}}{\mathrm{~d} z} \theta\right]-(\gamma-1) \frac{\mathrm{d} u_{z}}{\mathrm{~d} z}-\frac{\mathrm{d} \ln T_{0}}{\mathrm{~d} z} u_{z},
\end{aligned}\right.
$$

where $u_{z}$ denotes the vertical velocity, $\chi_{0}=K_{0} / \rho_{0} c_{\mathrm{p}}$ the radiative diffusivity, and the viscous dissipative term $\mathcal{D}_{v}$, which is given by

$\mathcal{D}_{v}=\frac{4}{3} v\left(\frac{\mathrm{d}^{2} u_{z}}{\mathrm{~d} z^{2}}+\frac{\mathrm{d} \ln \rho_{0}}{\mathrm{~d} z} \frac{\mathrm{d} u_{z}}{\mathrm{~d} z}\right)$.

This system (35) can formally be written as a generalised eigenvalue problem

$A \psi=\lambda B \psi$

where $\lambda=\tau+\mathrm{i} \omega$ is the complex eigenvalue associated with the eigenvector $\psi=\left(R, u_{z}, \theta\right)^{T}$, while $A, B$ denote differential operators.

Finally, the set of boundary conditions (7) written for $\left(u_{z}, \theta\right)$ is

$\left\{\begin{array}{l}u_{z}=0 \text { for } z=[0,1] \\ \frac{\mathrm{d} \theta}{\mathrm{d} z}+\frac{\mathrm{d} \ln T_{0}}{\mathrm{~d} z} \theta=0 \text { for } z=0 \\ \theta=0 \text { for } z=1 .\end{array}\right.$

\subsection{The numerical methods}

We solve the two linear algebra problems (30) and (37), (38) using the LSB code (Linear Solver Builder, Valdettaro et al. 2007). Both problems are discretised on the Gauss-Lobatto grid, which 
is associated with Chebyshev's polynomials; it leads to two distinct numerical problems:

1. The equilibrium model: the computation of equilibrium structure requires the solution of a nonlinear problem; this can be attempted using the so-called "Picard's method", which is based on the fixed-point algorithm (Hairer et al. 1993; Fukushima 1997), and involves solving our set of firstorder ordinary differential equations by successive iterations, that is, using the approach

$$
A \psi_{n+1}=B\left(\psi_{n}\right) \text {. }
$$

This scheme converges efficiently provided that the initial guess $\psi_{0}$ is not "too far" from the solution and that nonlinearities are weak.

2. The eigenmodes: we compute the entire spectrum of complex eigenvalues $\lambda$ using the QZ algorithm (Moler \& Stewart 1973), or compute a pair $(\lambda, \psi)$ about a given value of $\lambda$ using the iterative Arnoldi-Chebyshev algorithm (Arnoldi 1951; Saad 1992).

\section{Results}

In Eq. (32), we showed that the temperature contrast across the layer (and the associated contrasts of both density and pressure) is limited by the gravity field $g$. It would however be judicious not to restrict ourselves to small density contrasts because the mass involved between the conductivity hollow and the surface, that is $\Delta m$, plays a role in one of the two favourable criteria for the instability, see Eq. (21). We consider in the following a "convenient" value $g=7$.

We keep the radiative conductivity as small as possible because we would like to avoid too large values of diffusivity $\chi_{0}$, that is, we must not deviate significantly from the quasi-adiabatic approximation. We therefore choose $K_{\max }=10^{-2}$. The system (29), when combined with Schwartzschild's criterion (32), restricts the possible values of imposed bottom flux, and we therefore set $F_{\text {bot }}=2 \times 10^{-2}$ in the following. Using mild spatial resolutions, we choose a conservative value for the kinematic viscosity, that is, $v=5 \times 10^{-4}$.

\subsection{Computation of equilibrium fields}

To compute the equilibrium fields from the second-order system (29), we must choose two different boundary conditions, one for temperature and one for pressure. Without loss of generality, we set $T_{0}=1$ at the top of the layer. Since we are interested to locate the transition region of the fundamental mode in the layer middle, Eq. (21) must be satisfied at this location. We have said already that $F_{\text {bot }}=2 \times 10^{-2}$ and $K_{\max }=10^{-2}$. The temperature is fixed by $F_{\text {bot }}, K_{\max }$, and its own boundary condition. The pulsation period of the fundamental mode can be expressed by

$\Pi \simeq \frac{2 d}{\left\langle c_{\mathrm{s}}\right\rangle}$,

where $\left\langle c_{\mathrm{s}}\right\rangle$, which denotes the mean sound speed, is related to the temperature contrast across the layer. Thus, the only parameter that we can change in Eq. (21) is $\Delta m$. This value is directly linked to the boundary condition on density. To have a suitable $\Delta m$ value in the box middle, we therefore decide to take $\rho_{0}=2.5 \times$ $10^{-3}$ at the top of the layer (corresponding to $\ln P_{0}=-6.9$ ).

After deciding the values of the hollow parameters $T_{\text {bump }}, \mathcal{A}, e$ and $\sigma$-, we solve the problem (29) on the GaussLobatto grid and obtain the three equilibrium fields $T_{0}, P_{0}$ and

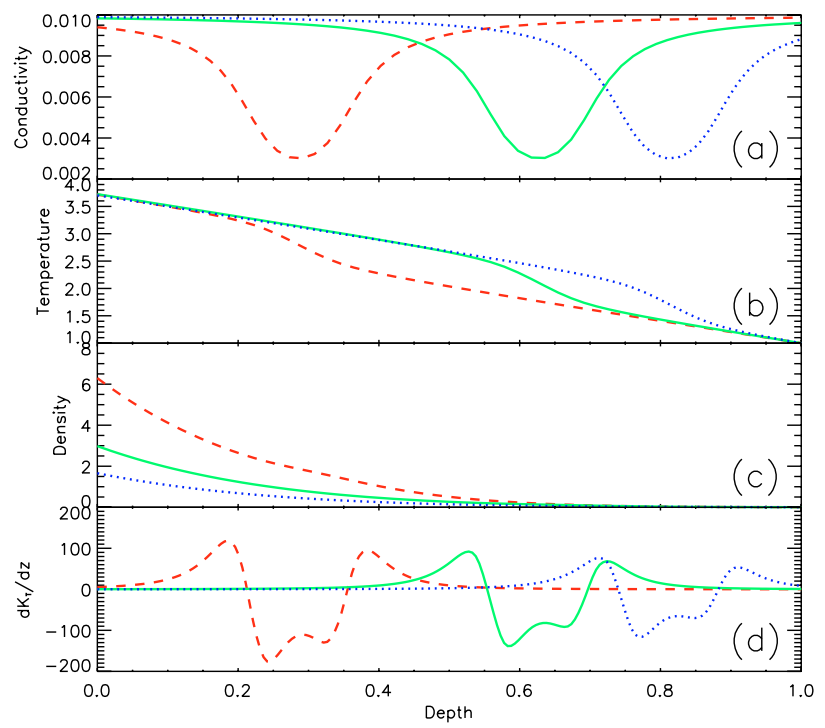

Fig. 3. a) Three different conductivity hollows: $T_{\text {bump }}=2.8$ (dashed red line), $T_{\text {bump }}=2.1$ (solid green line) and $T_{\text {bump }}=1.7$ (dotted blue line), with $\mathcal{A}=70 \%, e=0.4$ and $\sigma=7$; b) corresponding temperature profiles; c) density profiles; d) equilibrium profiles for $\mathrm{d} \mathcal{K}_{T} / \mathrm{d} z$.

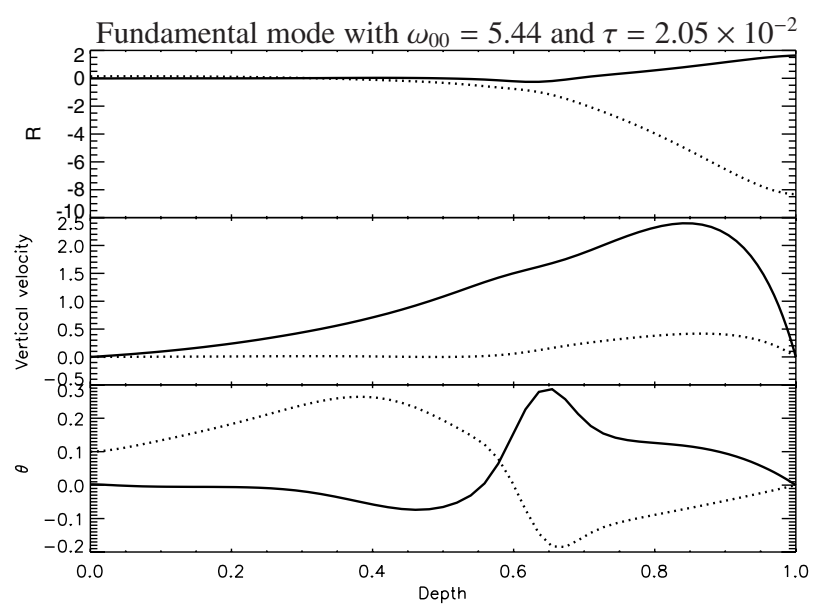

Fig. 4. Eigenfunctions $\left(R, u_{z}, \theta\right)$ for the unstable fundamental mode, corresponding to a hollow with $T_{\text {bump }}=2.1, \mathcal{A}=70 \%, e=0.4$ and $\sigma=7$. The equilibrium setup used to compute this mode is the one displayed by a solid green line in Fig. 3 .

$\rho_{0}$. Using these fields, we can compute any physical quantities required in the oscillations Eqs. (35), e.g. $\mathcal{K}_{T}$ or the diffusivity $\chi_{0}$. Some examples of such equilibrium fields derived are provided in Fig. 3, for three different positions of the conductivity hollow.

\subsection{Computation of eigenmodes}

Using the different equilibrium quantities, we solve the eigenvalue problem (35) to derive an entire spectrum of eigenvalues $\lambda$. Using the iterative Arnoldi-Chebyshev algorithm, we can obtain the closest eigenvalue using a close-guess value. In Fig. 4, we demonstrate how to derive the eigenfunctions of the unstable fundamental mode.

To check the convergence of this mode, we compute the spectra corresponding to the different eigenfunctions, as illustrated in Fig. 5. 


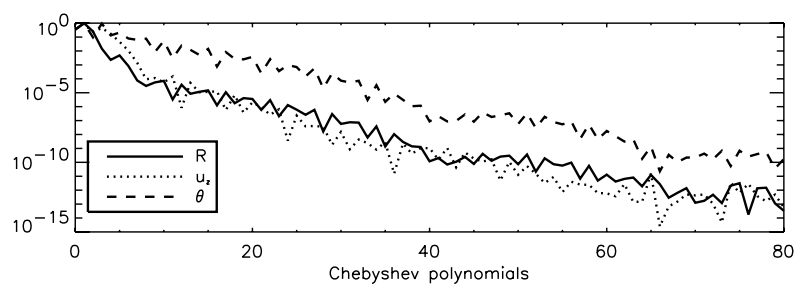

Fig. 5. Chebyshev's spectra of the different fields $\left(R, u_{z}, \theta\right)$ for the eigenmode shown in Fig. 4. Spectral precision is reached for every field.

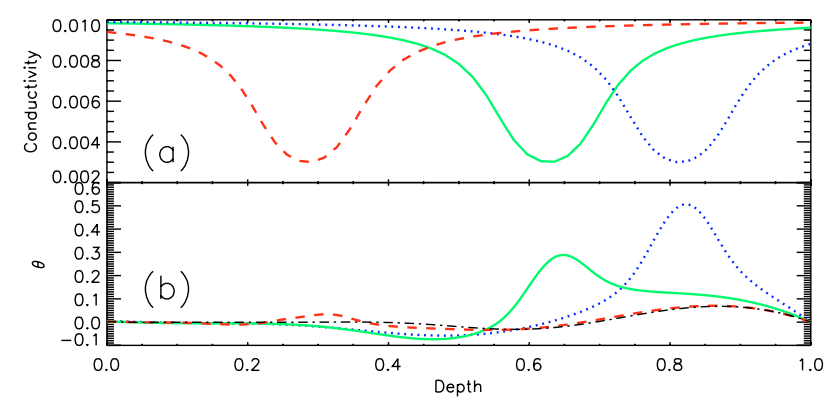

Fig. 6. a) Three different conductivity profiles with $T_{\text {bump }}=1.7$ (dotted blue line), $T_{\text {bump }}=2.1$ (solid green line) and $T_{\text {bump }}=2.8$ (dashed red line); b) corresponding fundamental eigenmodes (here the real part of temperature perturbations $\theta$ ). The case with a constant radiative conductivity $K_{0}\left(T_{0}\right)=K_{\max }$ is superimposed as a dot-dashed black line.

Finally, to determine the influence of the conductivity hollow on that fundamental eigenvector, we compute three eigenmodes using the different values of $T_{\text {bump }}$ used previously in Fig. 3. The result is displayed in Fig. 6 for a temperature perturbation $\theta$, where a constant radiative conductivity profile (i.e. without a conductivity hollow) is overplotted:

- The first case, corresponding to the dotted (blue) line, denotes a hot star in which the ionisation region is close to the surface. The conductivity hollow has an influence on the temperature eigenfunction, which is strongly deformed at its location. We show in Sect. 5.4 that this deformation is unable to destabilise this mode because the $\Psi$-criterion should also be taken into account (Eq. (21)).

- The second case, corresponding to the solid (green) line, highlights a case where ionisation occurs, to a first approximation, in the middle of the layer. The eigenvector is deformed by the conductivity hollow.

- The last case, which is indicated by the dashed (red) line, denotes a cold star in which the ionisation region is far from the surface. For this hollow location, the dynamical timescale is small compared to the local thermal one; thermodynamic transformations are therefore quasi-adiabatic, and non-adiabatic effects are negligible. This is confirmed in Fig. $6 \mathrm{~b}$ where the eigenmode has not been deformed by the conductivity hollow. To highlight this result, we superimpose the results for the case of a constant radiative conductivity $\left(K_{0}\left(T_{0}\right)=K_{\max }\right)$. The temperature perturbations (the dot-dashed (black) line) are close to the dashed (red) one, which means that the hollow has a marginal effect on mode stability.

We show that the conductivity hollow has an influence on the shape of the eigenmode, in the upper parts of our layer only. At greater depths, adiabatic thermodynamic transformations have an overwhelming effect, and the eigenvector is almost unchanged by variations in the conductivity profile.

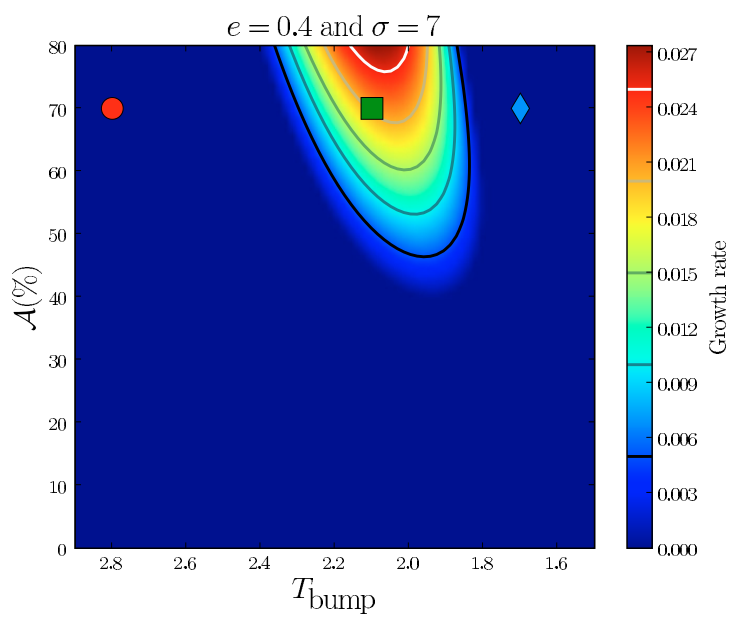

Fig. 7. Instability strip for the fundamental mode in the plane ( $T_{\text {bump }}, \mathcal{A}$ ) for given values of $e$ and $\sigma$ (isocontours in growth rates $\tau$ are displayed). The three marks correspond to the three particular computations done in Figs. 3 and 6 for $T_{\text {bump }}=1.7$ (blue diamond, stable), $T_{\text {bump }}=2.1$ (green square, unstable) and $T_{\text {bump }}=2.8$ (red circle, stable).

\subsection{Parametric surveys of the instability}

As claimed in Sect. 4.1, our conductivity profile is ideal for a parametric study of the $\kappa$-mechanism, because its parameters $T_{\text {bump }}, \mathcal{A}, e$, and $\sigma$ can be varied easily. We introduce the three parametric surveys that enable instability strips associated with the $\kappa$-mechanism, to be derived.

\subsubsection{The $T_{\text {bump }}-\mathcal{A}$ survey}

We want to investigate the influence of the hollow amplitude on stability. We fix a value of $\sigma$ and $e$, and vary the values of $T_{\text {bump }}$ and $\mathcal{A}$. For each value of these two parameters, we compute the equilibrium fields and then, the eigenvalues with their corresponding eigenvectors. Unstable modes are kept for all eigenvalues because their growth rate are positive. Figure 7 displays the obtained instability strip:

- dark (blue) areas correspond to stable fundamental modes (i.e. with $\tau<0$ );

- coloured areas indicate unstable fundamental modes, which have growth rates that become larger when a increasingly lighter colour is used.

Two major results can be derived from this figure: (i) a particular region in our layer ( $T_{\text {bump }} \in[1.8,2.3]$ ) appears to favour the creation of unstable modes, that is, one recovers the concept of transition region discussed in Sect. 3.2; (ii) a minimal amplitude in the hollow is required to achieve an instability $\left(\mathcal{A}_{\min } \simeq 45 \%\right)$.

\subsubsection{The $T_{\text {bump }}-\sigma$ survey}

We study the influence of the slope $\sigma$ of the conductivity hollow on stability. We choose a value for the amplitude $\mathcal{A}$ and width $e$, and allow $T_{\text {bump }}$ and $\sigma$ to vary. As for the previous survey, we plot in Fig. 8 the isocontours in growth rates $\tau$ but now in the plane $\left(T_{\text {bump }}, \sigma\right)$.

We find the same kind of areas as in Fig. 7, that is, an instability strip where modes are unstable (e.g. the coloured region) embedded in large (dark) regions where modes are stable. Nevertheless, the influence of the slope $\sigma$ on stability is 


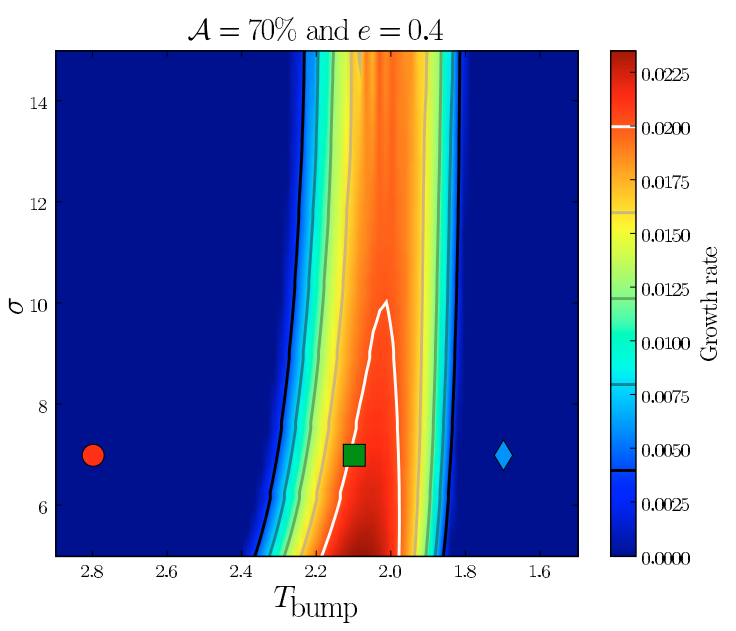

Fig. 8. Instability strip for the fundamental mode in the plane ( $\left.T_{\text {bump }}, \sigma\right)$, for given values of $\mathcal{A}$ and $e$. The blue diamond, green square and red circle correspond to the same modes displayed in Fig. 7.

less significant than that of amplitude. The instability strip covers the same temperature range as that presented in Fig. 7, $T_{\text {bump }} \in[1.8,2.3]$, but becomes almost vertical because there is no critical value of $\sigma$ to trigger destabilisation in the layer. In other words, the value of $\sigma$ is not affecting on the stability.

\subsubsection{The $T_{\text {bump }}-e$ survey}

Finally, we study the influence of the hollow width $e$ on the stability by performing a survey in the $\left(T_{\text {bump }}, e\right)$-plane, while keeping $\mathcal{A}$ and $\sigma$ constant. Results are displayed in Fig. 9.

An instability strip, which is similar to that shown in Fig. 7, is clearly visible; a minimal value exists for the width $e$, from which the fundamental mode becomes unstable $\left(e_{\min } \simeq 0.15\right)$. This implies that narrow hollows are insufficient to initiate the instability.

\subsubsection{Summary of the surveys}

These three parametric studies enable the respective influence of each of the hollow parameters on the layer stability to be studied:

- In Figs. 7-9, we observe a particular range in $T_{\text {bump }}$, i.e. $T_{\text {bump }} \in[1.8,2.3]$, within which the fundamental mode is unstable. It defines an instability strip in temperature and corresponds to the particular area in the layer that we introduced before as the transition region.

- Only the amplitude and width of the hollow influence stability because we find critical values for both of them, i.e. $\mathcal{A}_{\text {min }} \simeq 45 \%$ and $e_{\min } \simeq 0.15$. This can be linked to the condition (13), which entails that a precise shape in the conductivity profile is required to obtain unstable modes. Moreover, the hollow slope $\sigma$ is not a key control parameter because it does not modify the shape of the instability strip while it varies in value.

We now determine the favourable conditions for instability occurs, using the work-integral formalism.

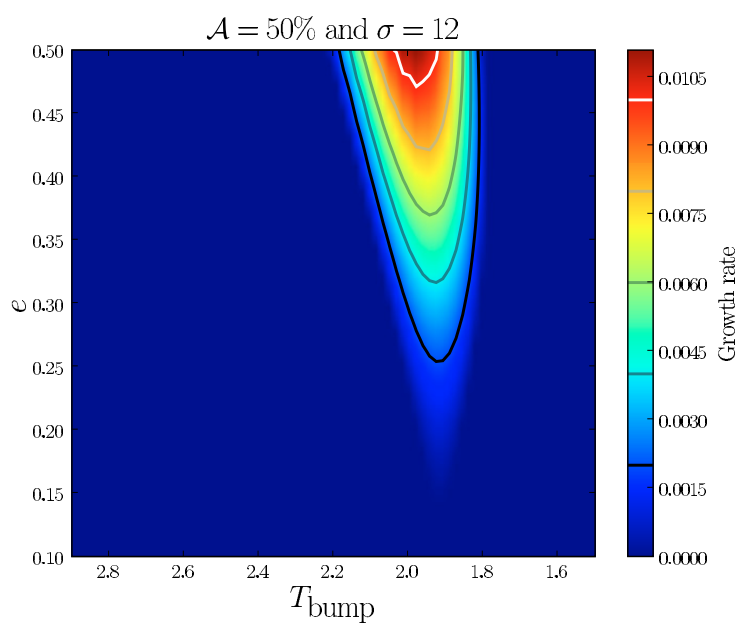

Fig. 9. Instability strip for the fundamental mode in the plane $\left(T_{\text {bump }}, e\right)$, for given values of $\mathcal{A}$ and $\sigma$.

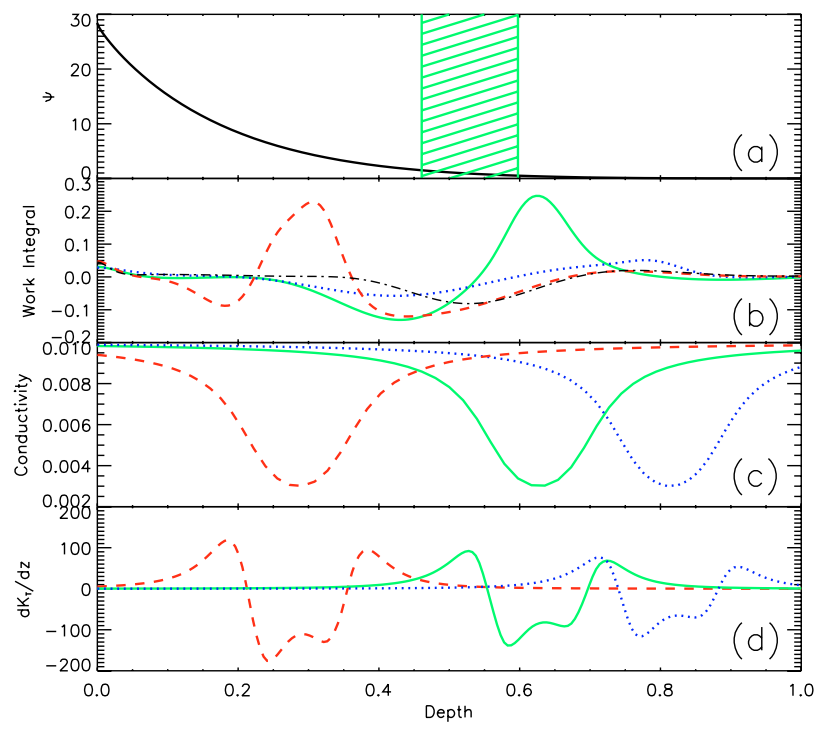

Fig. 10. a) Coefficient $\Psi$ plotted over the entire box (Eq. (20)). The green superimposed hatched zone represents the location where $\Psi=$ $1 \pm 0.5$. b) The real part of the work-integral numerator (Eq. (41)) plotted for the three different equilibrium models discussed already in Figs. 3 and 6. The case with a constant radiative conductivity is shown as a dot-dashed black line. c) Corresponding radiative conductivity profiles. d) Corresponding equilibrium field $\mathrm{d} \mathcal{K}_{T} / \mathrm{d} z$.

\subsection{Work integral in the non-adiabatic case}

Generalising the method described in Appendix A.1 to the nonadiabatic case, we obtain the following expression for the exact (complex) eigenvalue

$\lambda=\frac{\int_{0}^{1}\left[\frac{\gamma-1}{\gamma} T_{0}(\theta+R) \frac{\mathrm{d} u_{z}^{*}}{\mathrm{~d} z}-g R u_{z}^{*}+\mathcal{D}_{v} u_{z}^{*}\right] \rho_{0} \mathrm{~d} z}{\int_{0}^{1}\left|u_{z}\right|^{2} \rho_{0} \mathrm{~d} z}$

Because the denominator is always positive, the sign of the real part $\tau$ depends only on that of the numerator. To separate the regions of damping from that of driving, we plot the real part of the work integral in Fig. 10b.

The work integral is an invaluable tool because it enables the location of the driving to be determined precisely. The 
criteria provided in Eq. (22) predict that for a "sufficient" hollow, that is, of adequate amplitude and width, located in the transition region, the fundamental mode will be unstable. This result has already been confirmed using a parametric study (for example Fig. 7). Using the work integral, we consider the same three modes studied before in Figs. 3 and 7:

- The first case, plotted in dotted blue, corresponds to a hot star whose ionisation region is close to the surface. Since the density of the ionisation region is small, we therefore have $\Psi \ll 1$. In Fig. 10, we compare this case to that for a constant radiative conductivity, for which $K_{0}\left(T_{0}\right)=K_{\max }$ and $\mathrm{d} \mathcal{K}_{T} / \mathrm{d} z=0$; we conclude that the conductivity hollow has little influence on the work integral, that is that driving is unable to prevail over damping and $\tau<0$.

- In the second case, plotted in solid green, the radiative conductivity begins to decrease significantly at the location of the transition region where $\Psi \simeq 1$. As a consequence, driving becomes important (Eq. (22)). Furthermore, the radiative conductivity increase occurs where non-adiabatic effects are already significant, i.e. $\Psi<1$. This means that no damping will occur between the hollow position and the surface because the radiative flux perturbations are frozen-in. Driving is overcoming damping in this case and thus, $\tau>0$.

- The third case, plotted in dashed red, corresponds to a cold star whose ionisation region is located deeper in the stellar atmosphere where $\Psi \gg 1$. As shown in Fig. 6, ionisation occurs in a quasi-adiabatic location. As a consequence, excitation provided by the conductivity hollow cannot balance the damping arising in the layer, thus $\tau<0$.

In conclusion, Fig. 10 illustrates both conditions given in Eq. (22): (i) it is first necessary to have $\mathrm{d} \mathcal{K}_{T} / \mathrm{d} z<0$ to drive the oscillations. But this condition is insufficient to maintain the $\kappa$-mechanism; ( $i i)$ indeed, the thermal engine underlying the conductivity hollow has to be located neither too deep inside the star, nor too close to the surface. Therefore, only a specific range of effective temperatures allows the overlap of the transition and ionisation regions, which leads to the instability strips found in Figs. 7-9.

\section{Direct numerical simulations}

To confirm the reality of the instability strips discovered in the previous linear-stability analysis, we perform direct numerical simulations of the nonlinear problem. Starting from the favourable initial conditions determined by previous parametric surveys, we use nonlinear hydrodynamic equations to confirm:

- The onset of the instability sustained by the $\kappa$-mechanism and thus confirm the growth rates of the linear-stability analysis.

- The nonlinear saturation of the instability, which is of course not studied by the linear analysis, and have an estimation of the final amplitudes of modes.

All DNS were completed using the Pencil Code ${ }^{5}$. This nonconservative code is a high-order centered finite difference code (of the sixth order in space and third order in time) and conserved quantities are kept up to the discretization error of the scheme. On multiprocessor computers, it uses the MPI libraries (Message Passing Interface) which allow communications between processors and thus runs in parallel. Moreover, this code

\footnotetext{
5 See http://www.nordita.org/software/pencil-code/ and Brandenburg \& Dobler (2002).
}

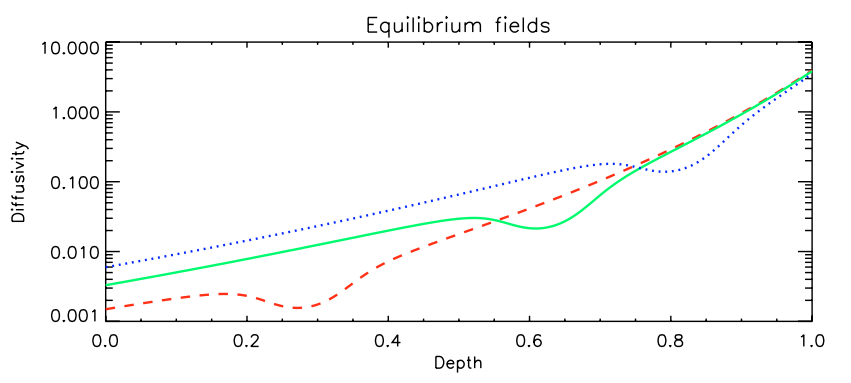

Fig. 11. Three different diffusivity profiles with $T_{\text {bump }}=1.7$ (dotted blue line), $T_{\text {bump }}=2.1$ (solid green line) and $T_{\text {bump }}=2.8($ dashed red line).

is fully explicit because the computation of the solution at time $t^{n+1}$ depends on the solution obtained at a time $t^{n}$ before. The timestep $\mathrm{d} t$ is therefore limited by an usual CourantFriedrichs-Levy (CFL) condition based on the consideration of the smallest physical timestep existing in the simulation as

$\mathrm{d} t \leq \min \left(c_{\delta t} \frac{\delta x}{c_{\mathrm{s}_{\max }}}, c_{\delta t \chi} \frac{\delta x^{2}}{\chi_{\max }}, c_{\delta t v} \frac{\delta x^{2}}{v_{\max }}\right)$,

where $c_{\delta t}, c_{\delta t \chi}$ and $c_{\delta t v}$ are constant coefficients depending on the spatial order of the scheme (see Sect. 19.2 in Press et al. 1992, hereafter PTVF92).

\subsection{The needed of an implicit solver}

This CFL condition is clearly a problem in our case because the most favourable setups correspond to large radiative diffusivities at the surface. Figure 11 emphasises this point where the diffusivity profile is plotted for the three common hollows used in this work $\left(T_{\text {bump }}=[1.7,2.1,2.8]\right)$. Because the $\Psi$-criterion (21) imposes a weak value of $\Delta m$ and hence of the top density, large surface values for the radiative diffusivity $\chi=K / \rho c_{\mathrm{p}} \simeq 3$ are obtained in all cases. This implies that the radiative diffusion timestep $c_{\delta t \chi} \delta x^{2} / \chi_{\max }$ in Eq. (42) is the smallest one and imposes a small timestep $\mathrm{d} t$. If we consider a typical spatial resolution of $256 \times 256$ (i.e. 256 gridpoints in each direction), we obtain $\mathrm{d} t \sim 10^{-6}$; in contrast, the dynamics of the layer is constrained by the sound speed, which has the dynamical timescale $\delta x / c_{\mathrm{s}_{\max }} \simeq 3 \times 10^{-3}$.

It is numerically prohibitive to reach the nonlinear saturation of excited acoustic modes with such an explicit solver. To limit the number of iteration, we decide to solve the diffusion of temperature implicitly. Because implicit schemes are unconditionally stable, we have no more constraints on the timestep from radiative diffusion and the CFL therefore becomes

$\mathrm{d} t \leq \min \left(c_{\delta t} \frac{\delta x}{c_{\mathrm{s}_{\max }}}, c_{\delta t v} \frac{\delta x^{2}}{v_{\max }}\right)$,

giving $\mathrm{d} t \sim 10^{-3}$ for the same resolution $256 \times 256$.

\subsection{The alternate direction implicit (ADI) scheme}

To solve the temperature equation, we adopt the time-split formulation provided in Malagoli et al. (1995). Firstly, we solve explicitly the three hydrodynamic equations, i.e. density, velocity, and temperature, but without solving the radiative diffusion term at this step. We then solve the temperature diffusion using the intermediate temperature $T_{\mathrm{expl}}^{n+1 / 2}$ as a source term. 
The time advance of the diffusion temperature equation is treated implicitly in the form

$\frac{T^{n+1}-T^{n}}{\mathrm{~d} t}=\frac{T_{\text {expl }}^{n+1 / 2}-T_{\text {expl }}^{n}}{\mathrm{~d} t}+\mathcal{R}$,

where the radiative diffusion term $\mathcal{R}$ is expressed by

$$
\begin{aligned}
\mathcal{R} & =\frac{1}{2} \frac{1}{\rho_{\operatorname{expl}}^{n+1 / 2} c_{\mathrm{v}}} \operatorname{div}\left[K\left(T^{n+1}\right) \boldsymbol{\nabla} T^{n+1}+K\left(T^{n}\right) \boldsymbol{\nabla} T^{n}\right] \\
& =\frac{1}{2}\left[\Lambda_{x}\left(T^{n+1}\right)+\Lambda_{x}\left(T^{n}\right)+\Lambda_{z}\left(T^{n+1}\right)+\Lambda_{z}\left(T^{n}\right)\right] .
\end{aligned}
$$

$\mathcal{R}$ could be modelled directly using a Cranck-Nicholson method, and a single matrix inversion with, for instance, a Successive Over Relaxation (SOR) method. For a 2D problem, this approach requires the inversion of a very sparse $N^{2} \times N^{2}$ matrix (e.g. Sect. 19.5 in PTVF92). We therefore implement an Alternate Direction Implicit (ADI) scheme that relies on operator splitting theory, which is frequently used in diffusion problems (e.g. Sect. 19.3 in PTVF92; Dendy 1977; Masalkar 1994). Solving implicitly both directions successively, we are able to consider tridiagonal matrices, or cyclic matrices depending on the boundary conditions used.

To treat implicitly the nonlinear terms (i.e. $K(T)$ ), we adopt the following approximation commonly referred to as Rosenbrock's method (Saarikoski et al. 1997; Witelski \& Bowen 2003)

$$
\Lambda\left(T^{n+1}\right)=\Lambda\left(T^{n}\right)+\underbrace{\left(\frac{\partial \Lambda}{\partial T^{n}}\right)}_{\equiv J}\left(T^{n+1}-T^{n}\right),
$$

where $J$ denotes the Jacobian matrix associated with the operator $\Lambda$. Expanding Eq. (44) in each direction, we obtain

$$
\left\{\begin{aligned}
\left(I-\frac{\mathrm{d} t}{2} J_{x}\right) \alpha & =\Lambda_{x}\left(T^{n}\right)+\Lambda_{z}\left(T^{n}\right)+\frac{T_{\text {expl }}^{n+1 / 2}-T_{\text {expl }}^{n}}{\mathrm{~d} t}, \\
\left(I-\frac{\mathrm{d} t}{2} J_{z}\right) \beta & =\alpha, \\
T^{n+1} & =T^{n}+\mathrm{d} t \cdot \beta
\end{aligned}\right.
$$

where $I, J_{x}$ and $J_{z}$ are the identity matrix, a cyclic matrix and a tridiagonal matrix, respectively.

\subsection{Results}

All 2D-simulations were completed using a mean resolution $16 \times 201$ and a constant kinematic viscosity $v=5 \times 10^{-4}$. To avoid the propagation of non-radial modes (Mulet-Marquis et al. 2007) as much as possible, we chose a "small" box with an aspect ratio $L_{x} / L_{z}=1$. If we refer to classical hydrodynamic instabilities, the critical horizontal wavelength $\lambda_{\text {crit }}$ from which instability develops is, in general, a bit larger than the vertical extent $L_{z}$ of the domain. As an example, $\lambda_{\text {crit }} / L_{z}=2 \sqrt{2}=2.83$ in the Rayleigh-Bénard convection (Chandrasekhar 1961) or $\lambda_{\text {crit }} / L_{z}=2.84$ in the compressible polytrope $m=1$ (Gough et al. 1976).

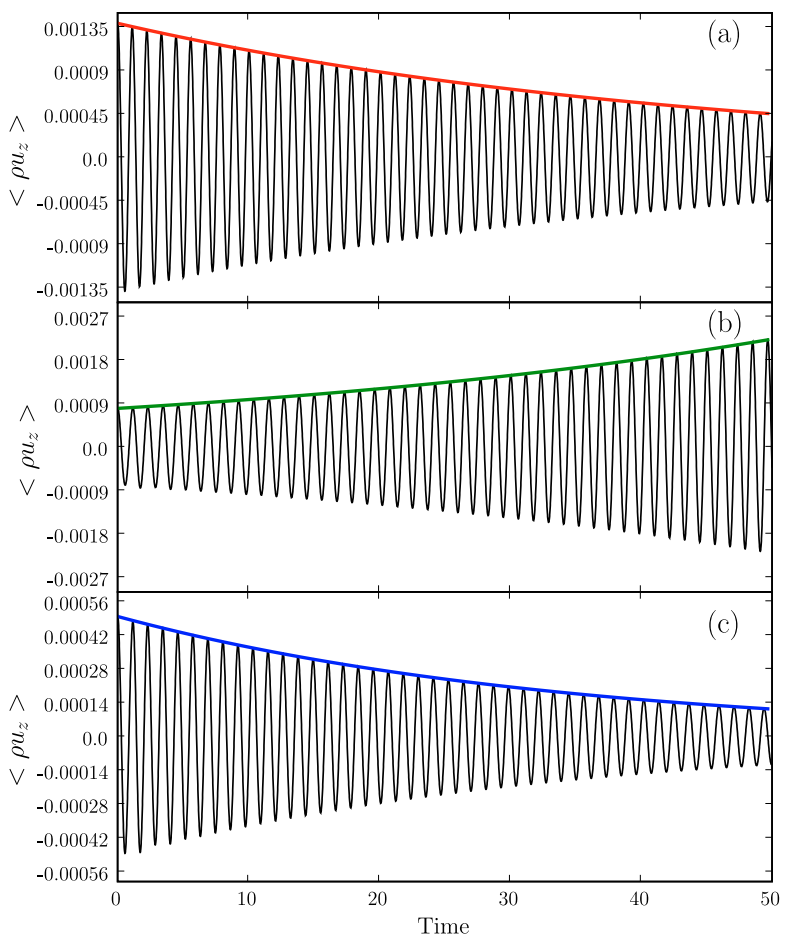

Fig. 12. The temporal evolution for times $t \in[0,50]$ of the mean vertical momentum $\left\langle\rho u_{z}\right\rangle$ with the three different starting setups. In each panel, the growing (or damping) curve calculated thanks to the linear-stability analysis is superimposed. a) $T_{\text {bump }}=2.8$ (red line). b) $T_{\text {bump }}=2.1$ (green line). c) $T_{\text {bump }}=1.7$ (blue line).

Table 1. Growth rates corresponding to the three equilibrium setups provided in Fig. 12 computed in the linear-stability analysis (the real part of eigenvalues) and in the DNS (computed from the exponential growth of the vertical momentum). The corresponding relative error is given.

\begin{tabular}{crrr}
\hline \hline & \multicolumn{1}{c}{$\tau_{\text {LSB }}$} & \multicolumn{1}{c}{$\tau_{\text {DNS }}$} & Rel. err. \\
\hline red & $-2.2415 \times 10^{-2}$ & $-2.2412 \times 10^{-2}$ & $1.5551 \times 10^{-4}$ \\
green & $2.0484 \times 10^{-2}$ & $2.0516 \times 10^{-2}$ & $1.5286 \times 10^{-3}$ \\
blue & $-2.9382 \times 10^{-2}$ & $-2.9339 \times 10^{-2}$ & $1.4877 \times 10^{-3}$ \\
\hline
\end{tabular}

\subsubsection{Growth rates from the DNS}

Figure 12 emphasises the temporal evolution of the mean momentum $\left\langle\rho u_{z}\right\rangle$ (where $\langle$. $\rangle$ is an average over the entire box) for the three common equilibrium setups (the red, green and blue ones corresponding respectively to $\left.T_{\text {bump }}=[2.8,2.1,1.7]\right)$. Only one case appears to be unstable because:

- The first (red) setup expresses the case of a cold star whose ionisation region is deep. The linear-stability analysis completed previously predicts that the excitation does not occur in this case (the red circle in Fig. 7 is well outside the instability strip). As seen in Fig. 12a, this result is confirmed by the nonlinear simulation because the mean vertical momentum decreases with time. Moreover, the damping rate calculated using LSB (indicated as a red line) is reproduced well by this simulation (Table 1 ).

- The second (green) setup denotes the case where the $\kappa$-mechanism is efficient. The fundamental $p$-mode is expected to be unstable (the green square inside the instability strip in Fig. 7). The DNS confirms this excitation (Fig. 12b) and the growth rate is the same as the predicted one (Table 1). 


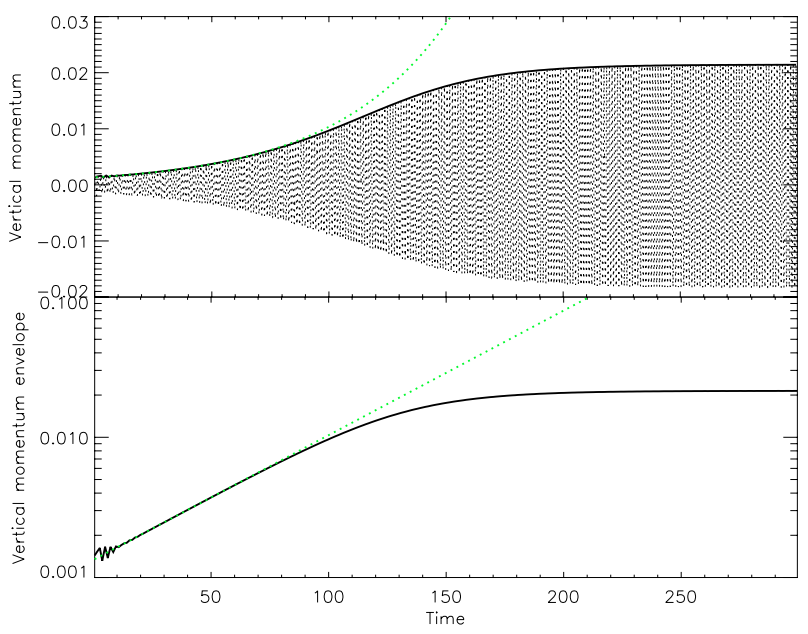

Fig. 13. Upper panel: temporal evolution of the mean vertical momentum $\left\langle\rho u_{z}\right\rangle$. The linear-stability analysis result from Fig. 4 is overplotted in green. Lower panel: temporal evolution of the corresponding envelope with the theoretical curve of growth still superimposed. Ordinate is scaled logarithmically.

- The third (blue) setup corresponds to a hot star whose ionisation is close to the surface. Damping phenomena prevail over excitation and the fundamental mode is stable (the blue diamond outside the instability strip in Fig. 7). The result is again confirmed by the DNS (see Fig. 12c and Table 1).

In Fig. 13, we integrate the DNS from the initial green setup with $T_{\text {bump }}=2.1$ until the approach towards the nonlinear limit cycle stability, that is, the nonlinear saturation. The (green) dotted line corresponds to the theoretical growth rate given in Table 1, that is, $\tau=2.0484 \times 10^{-2}$. The saturation of this mode appears to occur about time $t \simeq 200$, which corresponds approximately to 170 mode periods. Such a time interval is compatible with the characteristic timescale of instability given by $1 / \tau \sim 100$. Finally, the amplitude reached at the end of the saturation is about $\left\langle\rho u_{z}\right\rangle \simeq 2 \times 10^{-2}$. A careful study of mode saturation is beyond the scope of this paper and will be considered in future work.

\subsubsection{Vertical profiles}

The good agreement between the theoretical and DNS growth rates shown in Table 1 marks a first success in the reproduction of the $\kappa$-mechanism in our simulations. We address the modes structure by computing the vertical profiles from the DNS and comparing them to the eigenvectors of the linear-stability analysis.

In Fig. 14, we perform an horizontal and temporal Fourier transforms of the vertical velocity-field and plotted the resulting power spectrum at $k_{x}=0$ in the $(z, \omega)$-plane. Using this method, we are able to determine precisely which acoustic modes are excited and which not in our numerical experiment, as they emerge in this plane as "shark fin" peaks about certain frequencies (see Dintrans \& Brandenburg 2004). That is what is displayed in Fig. 14 where the radial acoustic mode with a frequency $\omega_{00} \simeq 5.44$ is excited and the agreement with the linear eigenfrequency is remarkable. The second overtone with $\omega_{02} \simeq 11.05$ also appears in the $(z, \omega)$-plane, and there continues to be a good agreement between the linear-stability analysis and the DNS. We note that this frequency is almost twice the value of the fundamental mode frequency. It implies that a resonance-like
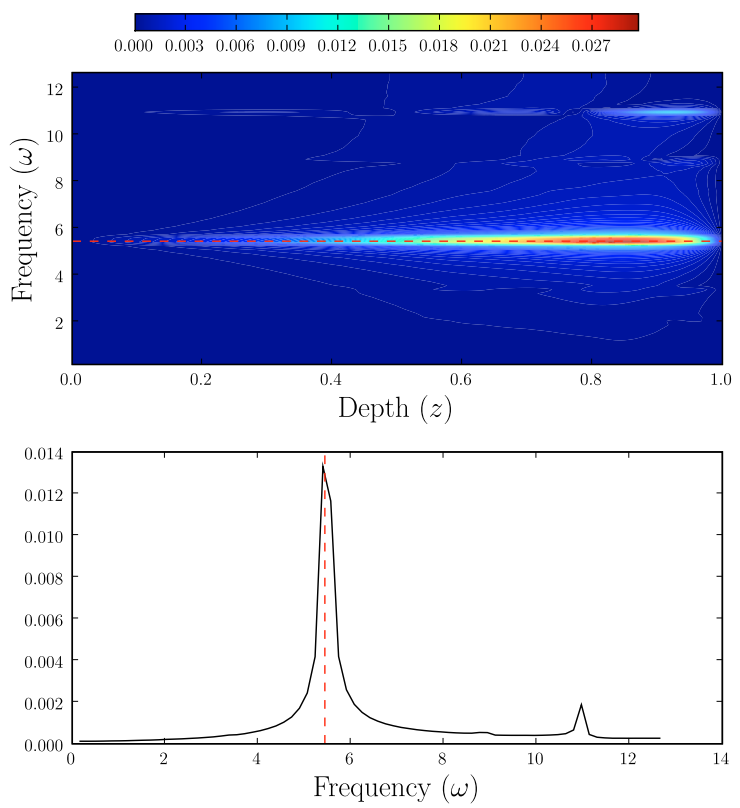

Fig. 14. Upper panel: temporal power spectrum in the $(z, \omega)$-plane for the radial modes $k_{x}=0$. Lower panel: the resulting spectrum after an integration in depth. Dotted lines correspond to the theoretical frequency $\omega_{00} \simeq 5.44$ obtained in the linear-stability analysis (e.g. Fig. 4).

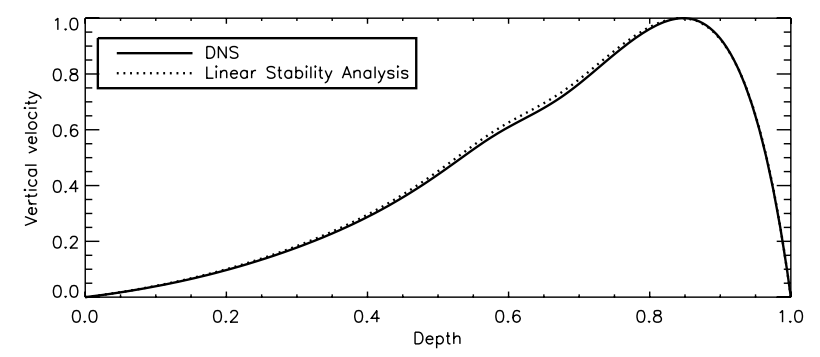

Fig. 15. Comparison between normalised vertical velocity profiles $u_{z}$ deduced from the DNS power spectrum (solid line) and the linear stability analysis (dashed line).

interaction occurs between these two modes: the $\kappa$-mechanism excites the fundamental mode, and a nonlinear interaction transfers energy between this mode and the second damped overtone, which leads to the nonlinear saturation.

We compare the eigenfunctions for given equilibrium setups, which were computed using LSB (see Fig. 4), to the vertical velocity $u_{z}$, which was measured using the power spectra shown in Fig. 4. These results are displayed in Fig. 15, which provides a mean profile computed about the frequency $\omega_{00}=5.44$. The DNS profile and the linear stability analysis profile overlap perfectly, which implies that we have agreement on the temporal (i.e. the frequency) and spatial (i.e. the pattern) scales of this mode.

\section{Conclusion}

We model the $\kappa$-mechanism in Cepheids by adapting methods that model a similar physical problem: the propagation of radial acoustic waves in a partially-ionised shell. Our model consists of a perfect gas embedded in an entirely radiative layer and the ionisation region is depicted by a configurable hollow in radiative conductivity. This approach enables both the shape and location of the ionised region in the layer to be altered, by changing the 
hollow parameters $T_{\text {bump }}, \mathcal{A}, \sigma$ and $e$ (Eqs. (25), (26)). We first check that a hollow of sufficient amplitude and width leads to

$\frac{\mathrm{d} \mathcal{K}_{T}}{\mathrm{~d} z}<0$,

which corresponds to the classical instability criterion derived using the work integral and quasi-adiabatic considerations. Another condition is however required to establish a thermal heat engine in the spirit of Eddington: the ionisation region must be located at a certain location in the layer, neither too deep nor too close to the surface. We show that this area is located in the so-called transition zone, which separates the quasi-adiabatic interior from the strongly non-adiabatic surface. This thermodynamical criterion can be summarised by

$T_{\text {ionisation }} \sim T_{\mathrm{TR}}$ and $\quad \frac{\left\langle c_{\mathrm{v}} T_{\mathrm{TR}}\right\rangle \Delta m}{\Pi L} \sim 1$.

Applying this second criterion, appropriate boundary conditions are chosen such that there is a transition region in the middle of our box for the fundamental acoustic mode. Both radiative and hydrostatic equilibria are discretised on the (spectral) Gauss-Lobatto grid and solutions are computed using a linear solver. We then perform a linear-stability analysis by computing the entire spectrum in addition to its associated eigenvectors for the radial-oscillation equations.

The main advantage of our approach is its ability to enable the parameters of the conductivity hollow to vary. The major achievement of the parametric surveys is a confirmation of the previous conditions, by means of the appearance of instability strips; only configurations that satisfy both conditions $(48,49)$ lead to unstable fundamental modes (e.g. Figs. 7 and 10).

These most favourable setups found in the linear-stability analysis are the starting points of our first 2D (nonlinear) DNS of $\kappa$-mechanism. Because of a too restrictive Courant-FriedrichsLevy (CFL) condition at the surface, these setups are not, however, suitable for a fully-explicit code such as the Pencil Code. We therefore develop a new implicit module to deal with the radiative-diffusion term and thus soften the CFL constraint. These DNS confirm both the growth rates and structures of linearly-unstable modes (see Figs. 13 and 15). Moreover, we are able to reach the nonlinear saturation that involves an intricate coupling between the fundamental mode and (at least) the damped second overtone of which the period is a multiple of the fundamental one.

This work constitutes a first step in our Cepheids' project devoted to the modelling of the convection-pulsation interaction in the coldest Cepheids, which are close to the red edge of the instability strip. It is well known that convection occurs in a nonnegligible fraction of these stars and modifies their pulsation properties. For now, models based on time-dependent convection theories reproduce well the position of this red edge, observational periods, and light curves (YKB98; Bono et al. 1999). However, they involve many free parameters that are either fitted to the observations or hardly constrained by theoretical values, which leads to almost identical results for different parameter sets (Kolláth et al. 2002; Szabó et al. 2007).

Despite their limitations (weak contrasts in pressure throughout the computational domain and thermal timescale problem, see e.g. Brandenburg et al. 2000), DNS are without a doubt a good way to address this convection-pulsation interaction because they fully account for the crucial nonlinearities. With a conductivity profile solely based on temperature (Eq. (25)), it is easy to fit locally the profile shape of one (or several) convective zone by increasing the temperature gradient above the adiabatic one. Indeed, as an ionisation region corresponds to a local increase in opacity, it is well known that convection can develop. This occurs in cold Cepheids where two separate convectivelyunstable regions coexist with $\mathrm{HeI} / \mathrm{H}$ and $\mathrm{HeII}$ ionisation regions. By adjusting in DNS the convective zone width or the strength of gravity, it will be possible to match the (local) turnover timescale of convection with the mean mode period, and then to study the coupling between convection and pulsation.

Acknowledgements. Calculations were carried out on the CalMip machine of the Centre Interuniversitaire de Calcul de Toulouse (CICT) which is gratefully acknowledged. It is also a pleasure to thank Isabelle Baraffe, Fabien Dubuffet, Marie-Jo Goupil and Michel Rieutord for their fruitful comments.

\section{Appendix A: Work integral}

\section{A.1. Using the quasi-adiabatic approximation}

In the energy equation in Syst. (3), we can first separate adiabatic terms from non-adiabatic ones as

$T^{\prime}=T_{\text {adia }}^{\prime}-\frac{1}{\rho_{0} c_{\mathrm{v}} \lambda} \operatorname{div} \boldsymbol{F}^{\prime}$

and, by using the ideal gas equation of state (1),

$p^{\prime}=p_{\text {adia }}^{\prime}-\frac{\gamma-1}{\lambda} \operatorname{div} \boldsymbol{F}^{\prime}$

This equation corresponds to the pressure perturbation due to both adiabatic and non-adiabatic oscillations. The momentum equation in Syst. (3) then becomes

$$
\begin{aligned}
(\lambda+\delta \lambda)(\boldsymbol{u}+\delta \boldsymbol{u})= & \underbrace{-\frac{1}{\rho_{0}} \boldsymbol{\nabla} p_{\text {adia }}^{\prime}+\frac{\rho_{\text {adia }}^{\prime}}{\rho_{0}} \boldsymbol{g}}_{\text {Adiabatic part }} \\
& \underbrace{+\frac{1}{\rho_{0}} \frac{\gamma-1}{\lambda} \boldsymbol{\nabla}\left(\operatorname{div} \boldsymbol{F}^{\prime}\right)}_{\text {Non-adiabatic perturbation }} .
\end{aligned}
$$

This equation can formally be written as a generalised eigenvalue problem with a perturbation operator

$(A+\delta A)(\boldsymbol{\psi}+\delta \boldsymbol{\psi})=(\lambda+\delta \lambda) B(\boldsymbol{\psi}+\delta \boldsymbol{\psi})$,

where $\delta \lambda$ and $\delta \psi$ are caused by the perturbation operator $\delta A$ (here the non-adiabatic effects). $\delta \lambda$ can then be obtained from a first-order perturbation analysis equivalent to these done in quantum mechanics (e.g. Bender \& Orszag 1978; Dyson \& Schutz 1979)

$\delta \lambda=\frac{\left\langle\psi_{0} \mid \delta A \psi_{0}\right\rangle}{\left\langle\psi_{0} \mid B \psi_{0}\right\rangle}$

where the symbol $\langle\mid\rangle$ means the following dot product (e.g. Lynden-Bell \& Ostriker 1967)

$\left\langle\boldsymbol{f}_{1} \mid \boldsymbol{f}_{2}\right\rangle=\int_{V} \boldsymbol{f}_{1}^{*} \cdot \boldsymbol{f}_{2} \rho_{0} \mathrm{dV}$

We thus obtain the expression for the eigenvalue perturbation $\delta \lambda$

$\delta \lambda=\frac{\gamma-1}{\lambda} \frac{\int_{V} \boldsymbol{u}^{*} \cdot \boldsymbol{\nabla}\left(\operatorname{div} \boldsymbol{F}^{\prime}\right) \mathrm{d} V}{\int_{V}|\boldsymbol{u}|^{2} \rho_{0} \mathrm{~d} V}$. 
We can go a step further using

$\boldsymbol{u}^{*} \cdot \boldsymbol{\nabla}\left(\operatorname{div} \boldsymbol{F}^{\prime}\right)=\operatorname{div}\left(\boldsymbol{u}^{*} \operatorname{div} \boldsymbol{F}^{\prime}\right)-\operatorname{div} \boldsymbol{u}^{*} \operatorname{div} \boldsymbol{F}^{\prime}$,

of which the first term in the RHS vanishes due to the rigid boundaries for the velocity as

$\int_{V} \operatorname{div}\left(\boldsymbol{u}^{*} \operatorname{div} \boldsymbol{F}^{\prime}\right) \mathrm{d} V=\oiint_{S} \boldsymbol{u}^{*} \operatorname{div} \boldsymbol{F}^{\prime} \cdot \mathrm{d} \boldsymbol{S}=0$.

The second term in the RHS can also be simplified by using the continuity equation

$\operatorname{div} \boldsymbol{u}^{*}=-\lambda^{*} \frac{\delta \rho^{*}}{\rho_{0}}$

where $\delta \rho$ is the Lagrangian perturbation of density. We finally get the expression for the eigenvalue perturbation in the nonadiabatic case as

$\delta \lambda=-\frac{\int_{V}(\gamma-1) \frac{\delta \rho^{*}}{\rho} \operatorname{div} \boldsymbol{F}^{\prime} \mathrm{d} V}{\int_{V}|\boldsymbol{u}|^{2} \rho_{0} \mathrm{~d} V}$,

where we have assumed that the adiabatic eigenmode is purely imaginary, that is $\lambda=\mathrm{i} \omega$, and thus $\lambda^{*} / \lambda=-1$. We note that $\mathfrak{R}(\delta \lambda)$ corresponds to the damping (or growth) rate of a nonadiabatic mode, commonly written $\tau$.

\section{A.2. A thermodynamical approach}

It is also possible to derive Eq. (A.11) by energetic considerations. Hereafter, we will essentially follow Hansen's demonstration (Hansen \& Kawaler 1994). Let us adopt a thin shell of mass $\mathrm{d} m$ at a certain radius. During a complete cycle of oscillations the work $\mathrm{d} W$ done by the shell on its surroundings is linked to the internal energy $\mathrm{d} U$ and heat $\mathrm{d} q$ gained by this shell through the first principle of thermodynamics

$\mathrm{d} q=\mathrm{d} U+\mathrm{d} W$.

Integrating over a cycle of oscillations leads to

$$
\oint \mathrm{d} q=\oint \mathrm{d} U+\oint \mathrm{d} W
$$

We are now going to suppose that the oscillation mechanism is quasi-adiabatic. It implies that every thin shell $\mathrm{d} m$ behaves as a Carnot-like heat engine where each process is reversible and the shell comes back to its initial position after each cycle of oscillations. Internal energy $\mathrm{d} U$ being a state variable, one gets $\oint \mathrm{d} U=0$ hence

$W=\oint \mathrm{d} q$.

Now one applies the second principle of thermodynamics claiming that

$\mathrm{d} S=\frac{\mathrm{d} q}{T}$.

After some time elapsed, we have $T=T_{0}+\delta T$ and then

$\mathrm{d} S=\frac{\mathrm{d} q}{T_{0}}-\frac{\delta T}{T_{0}^{2}} \mathrm{~d} q$, as a first-order approximation, with $\oint \mathrm{d} S=0$ for the same reason than $U$. We thus write

$\oint \mathrm{d} q=\oint \frac{\delta T}{T_{0}} \mathrm{~d} q$.

With Eqs. (A.14) and (A.17), the work over a cycle is given by

$W=\oint \frac{\delta T}{T_{0}} \mathrm{~d} q$

which leads to the total work integrated over mass shells

$W_{\text {tot }}=\int_{M} \oint \frac{\delta T}{T_{0}} \mathrm{~d} q \mathrm{~d} m$.

If $W_{\text {tot }}>0$, the star produces work over a cycle of oscillations and the initial perturbation will grow. In this case, the star is driving pulsation which is thus unstable. It may occur when shells gain heat (i.e. $\mathrm{d} q>0$ ) during compression phases (i.e. $\delta T>0)$ and this is the so-called "valve-mechanism" proposed by Eddington (1917).

Let us write the energy equation in the form

$\mathrm{d} q=-\frac{1}{\rho_{0}} \operatorname{div} \boldsymbol{F}^{\prime} \mathrm{d} t$,

and substitute it in Eq. (A.19)

$\frac{\mathrm{d} W_{\text {tot }}}{\mathrm{d} t}=-\int_{V} \frac{\delta T}{T_{0}} \operatorname{div} \boldsymbol{F}^{\prime} \mathrm{d} V$

Kinetic energy over a period of oscillations is given by

$E=\frac{1}{2} \int_{V}|\boldsymbol{u}|^{2} \rho_{0} \mathrm{~d} V$

and Eqs. (A.21), (A.22) lead to the following expression for the growth rate of a mode

$\tau=\frac{1}{2} \frac{\mathrm{d} W_{\mathrm{tot}} / \mathrm{d} t}{E}$

Note that the factor $1 / 2$ comes from the fact that the pulsation amplitude grows or decays one half as does energy. The two formulations Eqs. (A.11) and (A.23) are of course alike by assuming the quasi-adiabatic approximation $\delta T / T_{0} \simeq(\gamma-1) \delta \rho / \rho_{0}$.

\section{Appendix B: The radiative term in energy equation}

Radiative flux perturbations can be written as

$-\operatorname{div} \boldsymbol{F}^{\prime}=\operatorname{div}\left(K_{0} \boldsymbol{\nabla} T^{\prime}+K^{\prime} \boldsymbol{\nabla} T_{0}\right)$.

By assuming $\theta \equiv T^{\prime} / T_{0}$ and using Eq. (6), it entails

$-\frac{\mathrm{d}}{\mathrm{d} z} \boldsymbol{F}^{\prime}=\operatorname{div}\left(K_{0} T_{0} \frac{\mathrm{d} \theta}{\mathrm{d} z}+K_{0} \theta \frac{\mathrm{d} T_{0}}{\mathrm{~d} z}+K_{0} \mathcal{K}_{T} \theta \frac{\mathrm{d} T_{0}}{\mathrm{~d} z}\right) \boldsymbol{e}_{z}$.

We then expand $\mathcal{K}_{T}$ with equilibrium Eqs. (29) as

$\mathcal{K}_{T}=\frac{\mathrm{d} \ln K_{0}}{\mathrm{~d} \ln T_{0}}=-\frac{T_{0}}{F_{\text {bot }}} \frac{\mathrm{d} K_{0}}{\mathrm{~d} z}$,

and thus obtain

$-\operatorname{div} \boldsymbol{F}^{\prime}=\frac{\mathrm{d}}{\mathrm{d} z}\left(K_{0} T_{0} \frac{\mathrm{d} \theta}{\mathrm{d} z}+K_{0} \theta \frac{\mathrm{d} T_{0}}{\mathrm{~d} z}+\theta T_{0} \frac{\mathrm{d} K_{0}}{\mathrm{~d} z}\right)$.

Finally, one gets

$-\operatorname{div} \boldsymbol{F}^{\prime}=\frac{\mathrm{d}^{2}}{\mathrm{~d} z^{2}}\left(K_{0} T_{0} \theta\right)=\Delta_{z}\left(K_{0} T_{0} \theta\right)$. 


\section{References}

Arnoldi, W. E. 1951, QApMa, 9, 17

Baker, N., \& Kippenhahn, R. 1962, Z. Astrophys., 54, 114

Baker, N., \& Kippenhahn, R. 1965, ApJ, 142, 868

Bender, C. M., \& Orszag, S. A. 1978, Advanced Mathematical Methods for Scientists and Engineers (New York: McGraw-Hill)

Böhm-Vitense, E. 1958, Z. Astrophys., 46, 108

Bono, G., Marconi, M., \& Stellingwerf, R. F. 1999, ApJS, 122, 167

Brandenburg, A., \& Dobler, W. 2002, CoPhC, 147, 471

Brandenburg, A., Nordlund, A., \& Stein, R. F. 2000, Geophysical \& Astrophysical Convection, ed. P. A. Fox, \& R. M. Kerr (New York: Gordon and Breach Science Publishers)

Buchler, J. R. 1997, in Variables Stars and the Astrophysical Returns of the Microlensing Surveys, ed. R. Ferlet, J.-P. Maillard, \& B. Raban, 181

Carson, T. R. 1976, ARA\&A, 14, 95

Chandrasekhar, S. 1961, Hydrodynamic and hydromagnetic stability, International Series of Monographs on Physics (Oxford: Clarendon)

Christensen-Dalsgaard, J. 2003, Lecture Notes on Stellar Oscillations (Physical and Astronomical Institute of Aarhus University)

Cox, J. P. 1958, ApJ, 127, 194

Cox, J. P. 1963, ApJ, 138, 487

Cox, J. P. 1980, Theory of stellar pulsation, Research supported by the National Science Foundation (Princeton, NJ: Princeton University Press), 393

Dendy, J. E. 1977, SJNA., 14, 313

Dintrans, B., \& Brandenburg, A. 2004, A\&A, 421, 775

Dyson, J., \& Schutz, B. F. 1979, Roy. Soc. Lond. Proc. Ser. A, 368, 389

Eddington, A. S. 1917, The Observatory, 40, 290

Fukushima, T. 1997, AJ, 113, 1909

Gautschy, A., \& Saio, H. 1996, ARA\&A, 34, 551

Gehmeyr, M., \& Winkler, K.-H. A. 1992, A\&A, 253, 101

Gilliland, R. L., Bono, G., Edmonds, P. D., et al. 1998, ApJ, 507, 818

Gough, D. O. 1977, ApJ, 214, 196

Gough, D. O., Moore, D. R., Spiegel, E. A., \& Weiss, N. O. 1976, ApJ, 206, 536

Hairer, E., Norsett, S. P., \& Wanner, G. 1993, Solving Ordinary Differential Equations I: Nonstiff Problems (Berlin, Heidelberg, New York: SpringerVerlag)

Hansen, C. J., \& Kawaler, S. D. 1994, Stellar Interiors. Physical Principles, Structure, and Evolution XIII, Figs. 3 1/2" diskette (Berlin, Heidelberg,
New York: Springer-Verlag), Also Astronomy and Astrophysics Library, 445, 84

King, D. S., \& Cox, J. P. 1968, PASP, 80, 365

Kolláth, Z., Buchler, J. R., Szabó, R., \& Csubry, Z. 2002, A\&A, 385, 932

Kuhfuß, R. 1986, A\&A, 160, 116

Lynden-Bell, D., \& Ostriker, J. P. 1967, MNRAS, 136, 293

Malagoli, A., Dubey, A., Cattaneo, F., \& Levine, D. 1995, A Portable and Efficient Parallel Code for Astrophysical Fluid Dynamics, [http://astro. uchicago.edu/Computing/0n_Line/cfd95/camelse.html]

Masalkar, P. J. 1994, Optik, 4, 168

Mihalas, D., \& Weibel Mihalas, B. 1984, Foundations of radiation hydrodynamics (New York: Oxford University Press)

Moler, C. B., \& Stewart, G. W. 1973, SJNA., 10, 241

Mulet-Marquis, C., Glatzel, W., Baraffe, I., \& Winisdoerffer, C. 2007, A\&A, 465, 937

Press, W. H., Teukolsky, S. A., Vetterling, W. T., \& Flannery, B. P. 1992, Numerical recipes in FORTRAN. The art of scientific computing (Cambridge: University Press), 2nd edn. (PTVF92)

Saad, Y. 1992, Numerical Methods for Large Eigenvalue Problems (New York: Halsted Press)

Saarikoski, H., Salmio, R. P., Saarinen, J., Eirola, T., \& Tervonen, A. 1997, OptCo, 134, 362

Seaton, M. J., \& Badnell, N. R. 2004, MNRAS, 354, 457

Stellingwerf, R. F. 1982, ApJ, 262, 330

Szabó, R., Buchler, J. R., \& Bartee, J. 2007, ApJ, 667, 1150

Unno, W. 1967, PASJ, 19, 140

Unno, W., Osaki, Y., Ando, H., Saio, H., \& Shibahashi, H. 1989, Nonradial oscillations of stars (Tokyo: University of Tokyo Press), 2nd edn.

Valdettaro, L., Rieutord, M., Braconnier, T., \& Fraysse, V. 2007, JCoAM, 205, 382

Vitense, E. 1953, Z. Astrophys., 32, 135

Witelski, T. P., \& Bowen, M. 2003, ApNM., 45, 331

Wuchterl, G., \& Feuchtinger, M. U. 1998, A\&A, 340, 419

Yecko, P., \& Kollath, Z. 1998, in A Half Century of Stellar Pulsation Interpretation, ed. P. A. Bradley, \& J. A. Guzik, ASP Conf. Ser., 135, 94 Yecko, P. A., Kolláth, Z., \& Buchler, J. R. 1998, A\&A, 336, 553 (YKB98) Zhevakin, S. A. 1953, Russ. A. J., 30, 161

Zhevakin, S. A. 1963, ARA\&A, 1, 367 